University of Texas at El Paso

\title{
DigitalCommons@UTEP
}

Open Access Theses \& Dissertations

2015-01-01

\section{Skin Detection in Hyperspectral Images}

Stephanie Sanchez

University of Texas at El Paso, smsanchez1992@gmail.com

Follow this and additional works at: https://digitalcommons.utep.edu/open_etd

Part of the Electrical and Electronics Commons

\section{Recommended Citation}

Sanchez, Stephanie, "Skin Detection in Hyperspectral Images" (2015). Open Access Theses \& Dissertations. 1151.

https://digitalcommons.utep.edu/open_etd/1151

This is brought to you for free and open access by DigitalCommons@UTEP. It has been accepted for inclusion in Open Access Theses \& Dissertations

by an authorized administrator of DigitalCommons@UTEP. For more information, please contact lweber@utep.edu. 


\title{
SKIN DETECTION IN HYPERSPECTRAL IMAGES
}

\author{
STEPHANIE MICHELLE SANCHEZ \\ Department of Electrical and Computer Engineering
}

APPROVED:

Miguel Velez-Reyes, Ph.D., Chair

Sergio Cabrera, Ph.D.

Tzu-Liang (Bill) Tseng, Ph.D.

Charles Ambler, Ph.D.

Dean of the Graduate School 


\section{Copyright C}

by

Stephanie Michelle Sanchez

2015 


\section{Dedication}

To small kids with big dreams 


\title{
SKIN DETECTION IN HYPERSPECTRAL IMAGES
}

\author{
by \\ STEPHANIE MICHELLE SANCHEZ
}

THESIS

Presented to the Faculty of the Graduate School of The University of Texas at El Paso

in Partial Fulfillment

of the Requirements

for the Degree of

MASTER OF SCIENCE

Department of Electrical and Computer Engineering THE UNIVERSITY OF TEXAS AT EL PASO

May 2015 


\section{Acknowledgements}

Thomas Edison once said, "I've not failed, I've just found 10,000 ways that won't work." I can honestly say this this quote sums up my experience as a graduate student. Because this journey would not have been possible without the help and support of several individuals, I'd like to take the time to acknowledge a few of them.

I am thankful for Dr. Miguel Velez-Reyes' time and guidance throughout this research project. Aside from being my advisor, I'd like to thank him for funding me throughout my graduate studies and helping me find the necessary funds to attend and present this research at the 2014 SHPE Conference and the 2015 SPIE DSS conference.

I'd like to thank Dr. Alina Zare from the University of Missouri at Columbia, Dr. Paul Gader from the University of Florida, Dr. Ion Marques and Dr. Manuel Graña from Universidad del País Vasco (UPV/EHU) for providing archival imagery.

I'd also like to thank my defense committee, professors, staff, all the individuals who at one point took part in UTEP's Sensor and Signal Analytics Research Group and my fellow peers for continuously challenging and motivating me.

Without God, my family and close friends this journey would have been something else. Thank you for listing to me when I needed someone to vent to, cheering me up when I was down, believing in me when I'd lost hope and helping me find my way back when I had lost sight of my goals and objectives. Thank you for all for your continuous support throughout this journey.

This material is based upon work supported by 2014 UTEP Provost's Summer Research Assistant Program, the UTEP Texas Instruments Foundation Scholarship Program for Graduate Students, and the UTEP ECE Department. Partial support was also received from the UTEP NSFSTEM Scholarship Program (NSF Grant 10-60-113). Any opinions, findings, and conclusions or recommendations expressed in this material are those of the author(s) and do not necessarily reflect the views of the National Science Foundation. Some of the equipment used in this research was purchased with funds provided by the University of Texas System STARS program. 


\begin{abstract}
Hyperspectral imagers collect information of the scene being imaged at close contiguous bands in the electromagnetic spectrum at high spectral resolutions. Hyperspectral images are organized in a data cube where the $\mathrm{x}$ and $\mathrm{y}$ axes contain spatial information and the $\mathrm{z}$-axis contains spectral information. The number of applications for these imagers has grown over the years as they are now used in various fields (i.e. agriculture, surveillance, chemical imaging, environmental, medical, etc.). Many algorithms are described in the literature for skin detection using color imagery. However increased detection accuracy, in particularly over cluttered backgrounds, and of small targets and in low spatial resolution systems can be achieved by taking advantage of the spectral information that can be collected with multi/hyperspectral imagers. The ultimate goal of our research work was the development of a human presence detection system over different backgrounds using hyperspectral imaging in the 400-1000nm region of the spectrum that can be used in the context of search and rescue operations, and surveillance in defense and security applications. The $400-1000 \mathrm{~nm}$ region is chosen because of availability of low cost imagers in this region of the spectrum. This thesis presents preliminary results in the use of combinations of normalized difference indices that can be used to detect regions of interest in a scene that can be used as a pre-processor in a human detection system. A new normalized difference ratio, the Skin Normalized Difference Index (SNDI) is proposed. Experimental results show that a combination the NDGRI+NDVI+SNDI results in a probability of detection similar to that of the NDGRI. However, the combination of features results in a much lower probability of false alarm.
\end{abstract}




\section{Table of Contents}

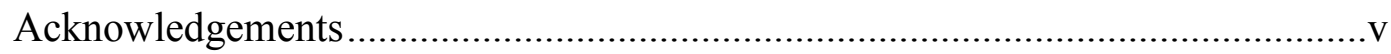

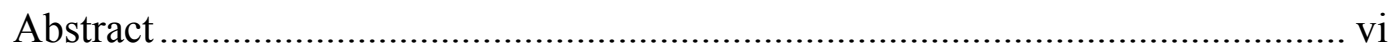

Table of Contents ................................................................................ vii

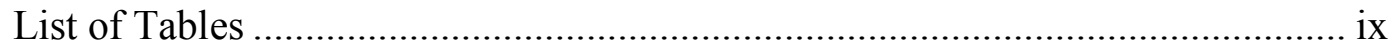

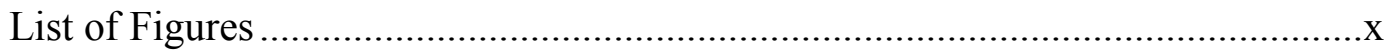

Chapter 1: Introduction .......................................................................... 1

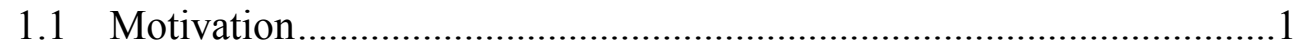

1.2 Research Objectives ................................................................ 1

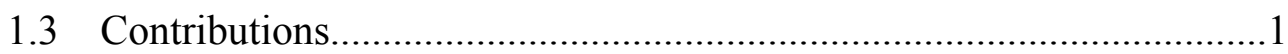

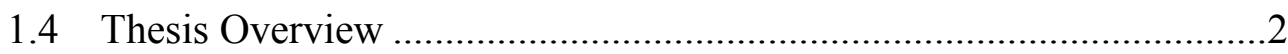

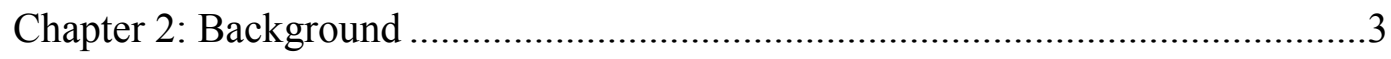

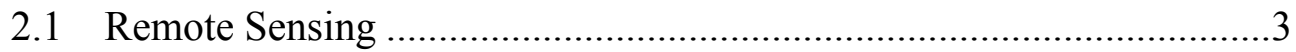

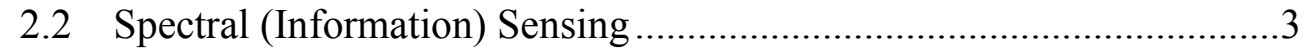

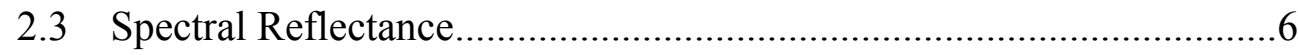

2. 4 Previous Research...................................................................... 11

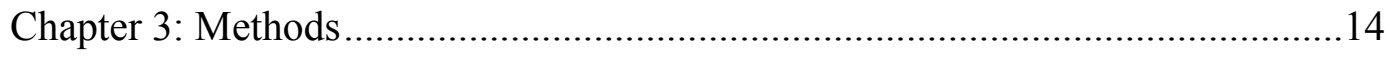

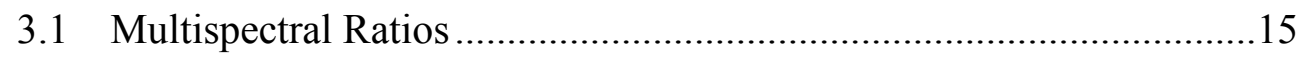

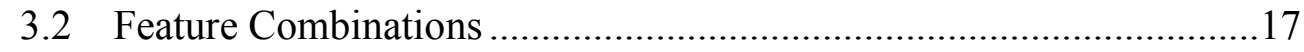

3.3 Hyperspectral Image Analysis Toolbox (HIAT) …..........................18

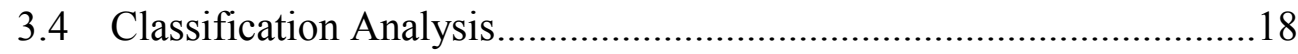

3.4 Hyperspectral Images used in the Experiments ..............................22

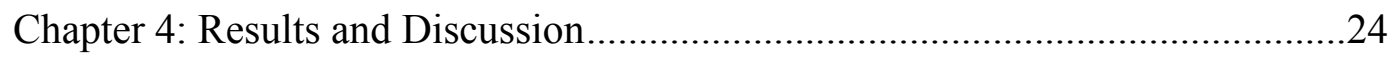

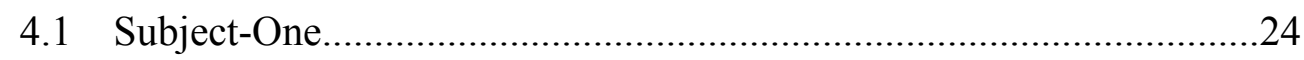

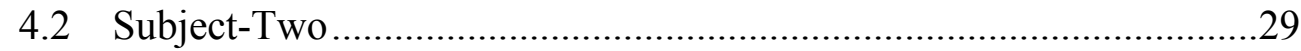

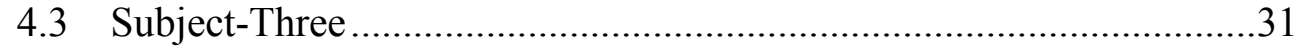

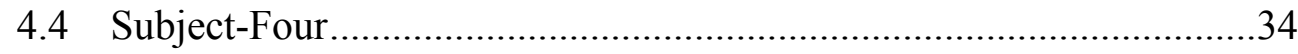

Chapter 5: Conclusion and Future Work ......................................................

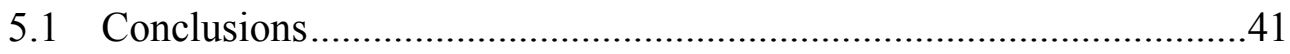




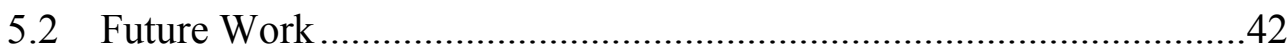

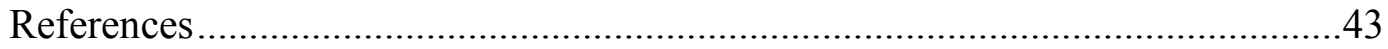

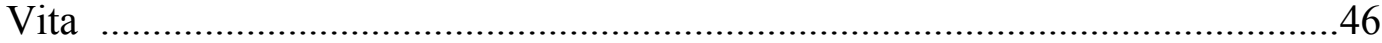




\section{List of Tables}

Table 1: Landsat 7 ETM+ Band Characteristic. ................................................................... 5

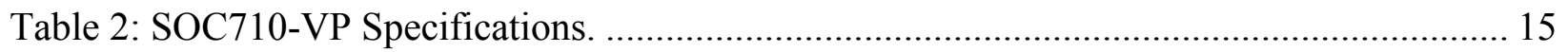

Table 3: Feature Combinations studied in the experiments................................................ 17

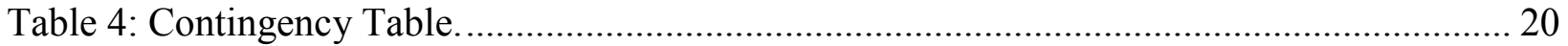

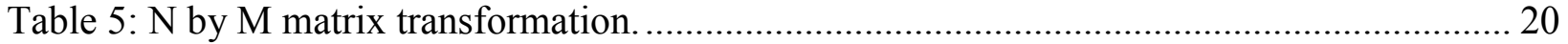

Table 6: Simplified Contingency Table and Accuracy Assessment...................................... 21

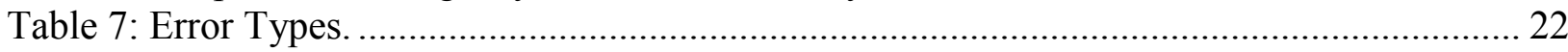

Table 8: Accuracy assessment for subject-one face image............................................... 28

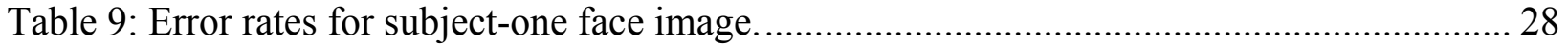

Table 10: Accuracy assessment for subject-two face image. ........................................... 31

Table 9: Error rates for subject-two face image............................................................ 31

Table 12: Accuracy assessment for subject-three face image. ............................................ 34

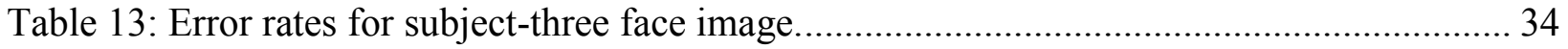

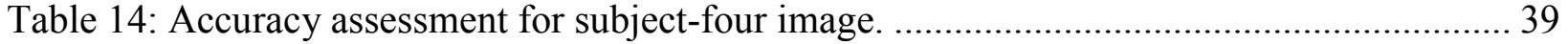

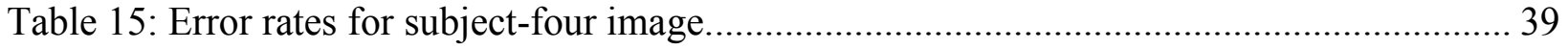




\section{List of Figures}

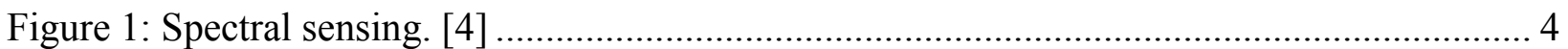

Figure 2: (a) Hyperspectral cube. (b) Pixel spectral signature. [6] ............................................ 6

Figure 3: Hyperspectral sensor platform and data collection. [7] ......................................... 6

Figure 4: Healthy leaf reflectance and primary absorption bands. [8] .................................. 7

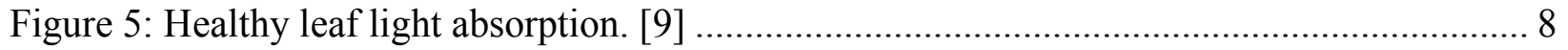

Figure 6: Vegetation reflectance. [11] ........................................................................... 9

Figure 7: The reflectance and absorption of the skin attributes to the skin coloration of a person.

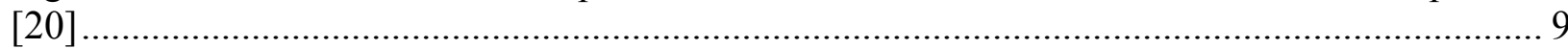

Figure 8: (a) Skin complexion based on the melanosomes percentage. (b) Spectral reflectance of

human skin at VIS-SWIR wavelengths [22] .................................................................. 10

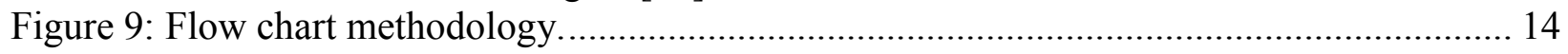

Figure 10: Subject one's testing and training polygons................................................. 19

Figure 11: True color RGB composites using R 650nm, G 510nm and B 475nm for each

subject. (a) Subject-One. (b) Subject-Two. (c) Subject- Three. (d) Subject-Four...................... 23

Figure 12: True color RGB composites with the corresponding testing and training polygons for

each subject. (a) Subject-One. (b) Subject-Two. (c) Subject-Three. (d) Subject-Four. ............... 24

Figure 13: Color palettes for (a) NDVI, (b) NDGRI, (c) SNDI, and (d) RGB composite using R-

NDGRI, G-NDVI and B-SNDI............................................................................... 25

Figure 14: Binary classification of skin and non-skin: I) Full HSI cube. II)

NDGRI+NDVI+SNDI. III) NDGRI+NDVI. IV) NDGRI + SNDI. V) NDVI+SNDI. VI)

$\begin{array}{ll}\text { NDGRI. VII) SNDI. } & 27\end{array}$

Figure 15: Color palettes for (a) NDVI, (b) NDGRI, (c) SNDI, and (d) RGB composite using R-

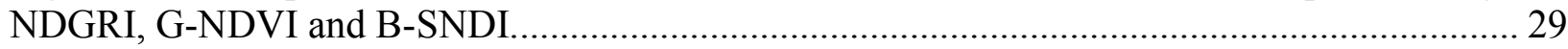

Figure 16: Binary classification of skin and non-skin: I) Full HSI cube. II)

NDGRI+NDVI+SNDI. III) NDGRI+NDVI. IV) NDGRI + SNDI. V) NDVI+SNDI. VI)

NDGRI. VII) SNDI. 30

Figure 17: Color palettes for (a) NDVI, (b) NDGRI, (c) SNDI, and (d) RGB composite using R-

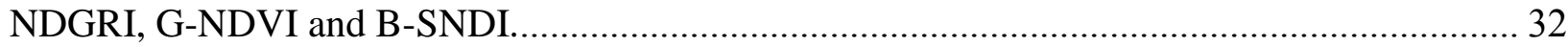

Figure 18: Binary classification of skin and non-skin: I) Full HSI cube. II)

NDGRI+NDVI+SNDI. III) NDGRI+NDVI. IV) NDGRI + SNDI. V) NDVI+SNDI. VI)

NDGRI. VII) SNDI.

Figure 19: Color palettes for (a) NDVI, (b) NDGRI, (c) SNDI, and (d) RGB composite using R-

NDGRI, G-NDVI and B-SNDI. RGB composites using R 650nm, G $510 \mathrm{~nm}$ and B 475nm... 35

Figure 20: Color palettes for (a) NDVI, (b) NDGRI, (c) SNDI, and (d) RGB composite using R-

NDGRI, G-NDVI and B-SNDI. RGB composites using R 650nm, G 510nm and B 475nm... 36

Figure 21: Binary classification of skin and non-skin: I) Full HSI cube. II)

NDGRI+NDVI+SNDI. III) NDGRI+NDVI. IV) NDGRI + SNDI. V) NDVI+SNDI. VI)

NDGRI. VII) SNDI.

Figure 22: Binary classification of skin and non-skin: I) Full HSI cube. II)

NDGRI+NDVI+SNDI. III) NDGRI+NDVI. IV) NDGRI + SNDI. V) NDVI+SNDI. VI)

NDGRI. VII) SNDI.

38 


\section{Chapter 1: Introduction}

This first chapter contains the motivation for conducting this project, the research objectives, and the overall contributions conducted. In addition, there is a brief overview of the thesis structure provided in the last section.

\subsection{Motivation}

Many algorithms are described in the literature for human skin detection in color imagery [1]. Increased detection accuracy, in particularly over cluttered backgrounds and in low spatial resolution systems, can be achieved by taking advantage of the spectral information that can be collected with hyperspectral imagers. With hyperspectral imaging emerging in different fields having vast amount of applications, defense and surveillance could greatly benefit from this technology. The benefit to utilizing hyperspectral imaging is the ability to detect skin over a cluttered background as every material has a unique spectral signature, including skin. Two examples of where skin detection in hyperspectral imaging may be applied are localizing a camouflaged sniper and finding human survivors after any type of natural disasters.

\subsection{Research Objectives}

The ultimate goal of our research work is the development of a human presence detection system over different backgrounds using hyperspectral imaging in the 400-1000 nm region of the electromagnetic spectrum. Such as system can be an important component in search and rescue operations, and surveillance applications in defense and security. The 400-1000 $\mathrm{nm}$ region is chosen because of availability of low cost hyperspectral imagers in this region of the spectrum from multiple vendors.

\subsection{Contributions}

In this thesis, we present experimental results comparing the performance of different normalized different indices (NDI) for skin detection. Due to their simplicity and robustness, NDI are attractive features to use in the pre-screening stage of a target detection system to identify 
regions of interest that can be further studied using more standard target detection techniques for target identification. In addition to utilizing literature proposed indices a new index was proposed, the Skin Normalized Difference Index (SNDI).

\subsection{Thesis Overview}

The contents of the thesis are structured as follows. Chapter two provides background on hyperspectral imaging, spectral properties of skin, and previous skin detection research for color and hyperspectral images. Chapter three contains the methodology section, with a brief review of each of the techniques utilized. Chapter four contains the experimental results and all corresponding observations after running the proposed Normalized Difference Indices. Chapter five contains the research's conclusions along with future work suggestions. 


\section{Chapter 2: Background}

With an increase in technology hyperspectral imaging has emerged in various application fields including surveillance and defense. In its early stages airborne images were taken as a source of information that allowed detection of objects and features. The Department of Defense (DoD) and the National Aeronautics and Space Administration (NASA) led to the development of many systems in the remote sensing area. The first class of remote sensors used in aerial photography, were monochrome and panchromatic cameras. Later in the 1960's the DoD created the first multispectral cameras, when they modified cameras with special spectral filters. The launch of Landsat 1, in July 1972 by NASA, was the first system capable of producing multispectral data in digital format. [2] Spectroscopy has come a long way since then increasing the number of defense and commercial applications. Osama bin Laden's capture, also known as Operation Neptune's Spear, where navigators used highly classified hyperspectral imagers aboard the MH-60 helicopters can be attributed to these improvements. [3]

\subsection{Remote Sensing}

The ability to obtain information about an object, area or phenomenon via data analysis acquired by a device that was never in contact with it is known as remote sensing. In order to perform remote sensing one must have a platform and a sensor. There are two types of remote sensing, active and passive. Active remote sensing requires the source to first emit a signal, while passive remote sensing uses existing energy that is reflected or emitted from a scene. Passive devices sources only detect emitted radiation by the objects being viewed or reflected by any other surrounding objects. Hyperspectral imagers and spectrometers are two passive remote sensing devices; both of these sources collect spectral information.

\subsection{Spectral (Information) Sensing}

The visible spectrum, classified as the electromagnetic spectrum detectable by the human eye, is about 30 to $700 \mathrm{~nm}$, which contains the average red $(\sim 620 \mathrm{~nm}$ to $780 \mathrm{~nm})$, green $(\sim 492 \mathrm{~nm}$ to 577 ) and blue ( $455 \mathrm{~nm}$ to $492 \mathrm{~nm}$ ) spectral sensors. Aside from RGB sensors, imagers may 
contain Near Infrared (NIR), Short-wave Infrared (SWIR), Mid-wave Infrared (MWIR), Longwave infrared (LWIR) are common wavelengths found in spectral sensors. Spectral sensing may be split into three major categories panchromatic, multispectral and hyperspectral imagers. These imagers are categorized from low to high spectral resolution quality. Figure 1 shows the common band wavelength found in multi/hyperspectral sensors and the sensor types.

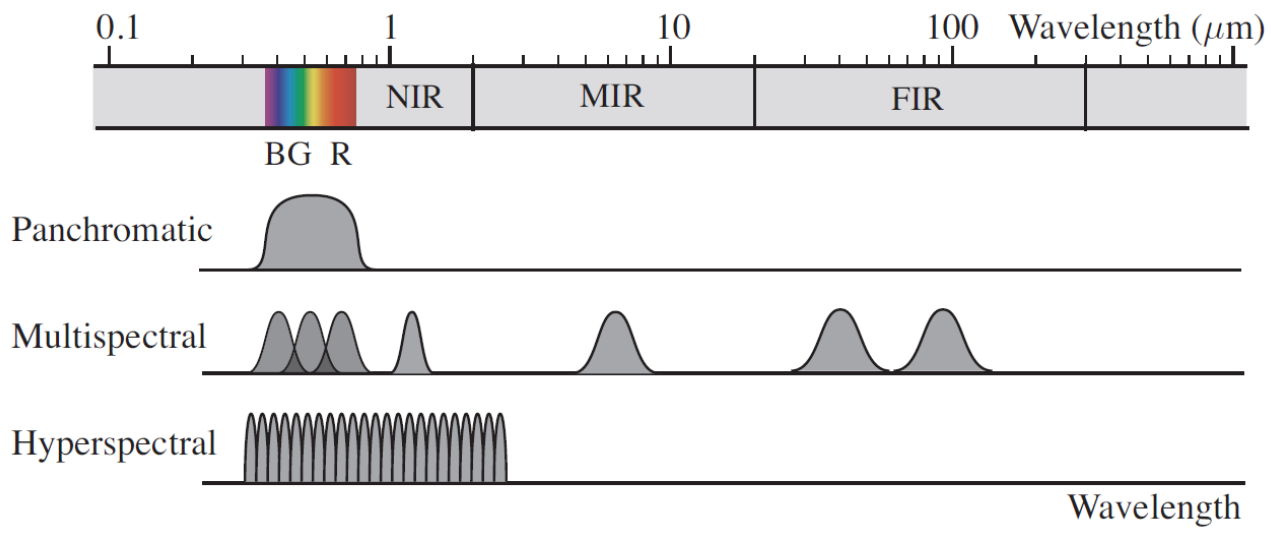

Figure 1: Spectral sensing. [4]

\subsubsection{Panchromatic Imagers}

Panchromatic imagers are only capable of collecting one band. These imagers are typically used in detection applications to identify the existence of materials, objects, activities, or events in a given area by their shape.

\subsubsection{Multispectral Imagers}

Multispectral imagers acquire several to tens of bands and have medium spectral resolution. These images are non-contiguous between their spectrum coverage, meaning all bands are not nearby one another which may be observed in both Figure 1 and Table 1. Landsat Enhanced Thematic Mapper Plus (ETM+), a multispectral imager, stores a panchromatic band and seven spectral bands ranging from blue wavelengths to thermal infrared. These imagers may be used for detection, classification and discrimination, as they are able to separate minerals into spectrally similar groups and generate generic categories of the foreign classes. 
Table 1: Landsat 7 ETM+ Band Characteristic.

\begin{tabular}{|c|c|c|c|}
\hline \multirow{9}{*}{ 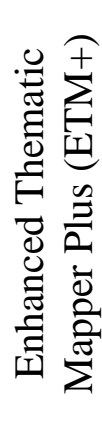 } & Landsat 7 & Wavelength $(\mu \mathrm{m})$ & Resolution (m) \\
\hline & Band 1 & $0.45-0.52$ & 30 \\
\hline & Band 2 & $0.52-0.60$ & 30 \\
\hline & Band 3 & $0.63-0.69$ & 30 \\
\hline & Band 4 & $0.77-0.90$ & 30 \\
\hline & Band 5 & $1.55-1.75$ & 30 \\
\hline & Band 6 & $10.40-12.5$ & 30 \\
\hline & Band 7 & $2.09-2.35$ & 30 \\
\hline & Pan & $0.52-0.90$ & 30 \\
\hline
\end{tabular}

\subsubsection{Hyperspectral Imagers}

Hyperspectral imaging (HSI) is a technology that provides fully registered spatial and highresolution spectral (radiance, reflectance, or emission) information of the scene in the field of view of the sensor [5]. Hyperspectral imagers have dozens to hundreds of narrow contiguous bands which may be observed in Figure 1. Hyperspectral imagers utilize reflected light or emitted light as the energy source. The illuminating source must provide a broadband source to ensure all wavelengths in the sensor are spectral range are energized, in other words we would not want to discriminate a random wavelength due to poor illumination conditions; for this reason fluorescent bulbs of any type are non-ideal. Under proper illumination conditions these imagers may be used to identify and characterize certain scene features based on spectral signatures.

Hyperspectral images are organized as a three-dimensional cube, shown in Figure 2, where the $\mathrm{x}$ and $\mathrm{y}$ plane record the spatial information and $\lambda$, the third dimension, contains the spectral reflectance.

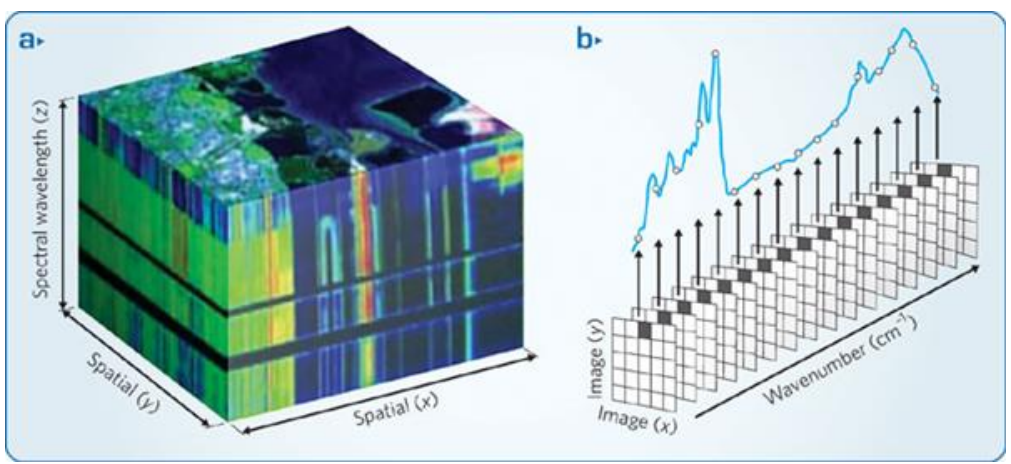


Figure 2: (a) Hyperspectral cube. (b) Pixel spectral signature. [6]

Hyperspectral imaging, originally developed for mining and geology to identify minerals, has spread into numerous fields ranging from surveillance to medical applications. As this technology is becoming more available to the public (i.e. research and academic institutions), organizations have posted various catalogued mineral/material compounds and their spectral signatures. Hyperspectral imaging provides images at multiple wavelengths to create a spectral signature for each pixel. Figure 3 displays a simple hyperspectral imaging platform along with some common average reflectance curves for cataloged materials.

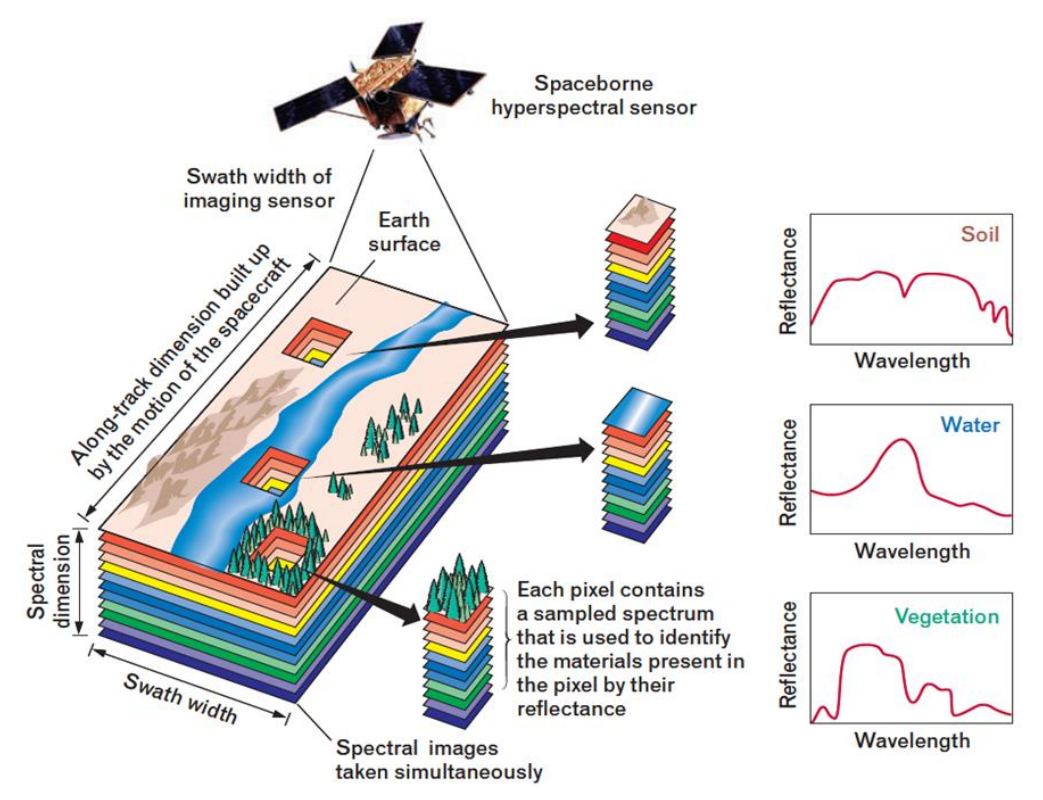

Figure 3: Hyperspectral sensor platform and data collection. [7]

\subsection{Spectral Reflectance}

Spectral reflectance, the ratio of reflected energy to incident energy as a function of wavelength, is the fundamental property wanted in reflected-light spectroscopy. Reflectance is a unit-less quantity that ranges from 0 to 1.0 or 0 to 100 when expressed as a percentage. Reflectance is obtained by dividing the reflected energy in a given wavelength by the incident energy. Reflectance varies at different wavelengths due to materials energy abortions and scattering. Spectral reflectance curves, plots of reflectance over wavelength, allow us to observe these 
variations. Peaks typically indicate reflectance values; the more reflective it is at a given wavelength the higher magnitude it has in its spectral signature. Similarly absorption features (i.e. downward deflections in the reflectance curve) indicate absorption values. The wavelength range for which a material absorbs the incident energy is known as an absorption band. The shape of a spectral signature along with the absorption bands strength and location allows for the identification and discrimination of materials. Figure 4 depicts how the absorption and reflection of an object varies depending on the dominant factors at different wavelengths.

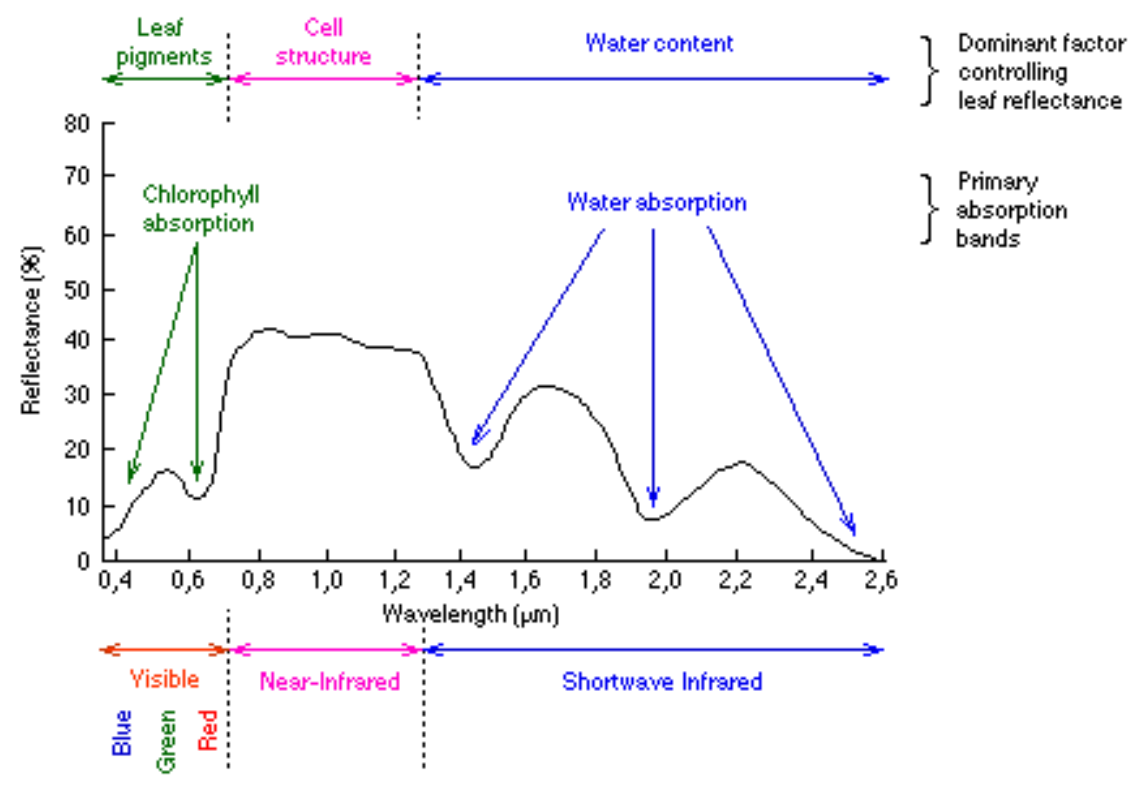

Figure 4: Healthy leaf reflectance and primary absorption bands. [8]

\subsubsection{Vegetation Spectra}

Vegetation's spectral reflectance has particular characteristics that may be observed throughout the spectrum. Figure 5 depicts the structure of a leaf and how it affects its reflectance. 


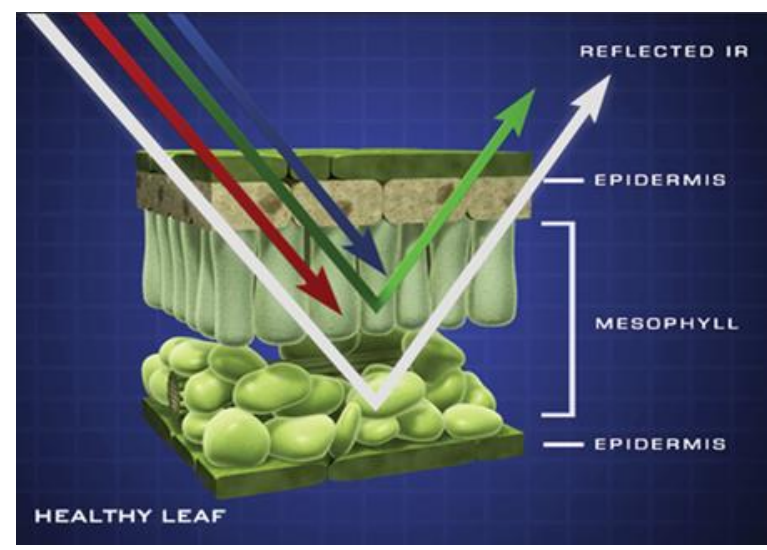

Figure 5: Healthy leaf light absorption. [9]

In the visible range the spectral shape is mainly dictated by chlorophyll and other leaf pigments' absorption effects. Chlorophyll, a biomolecule, may be found in cyanobacteria, chloroplasts of algae, and plants. This biomolecule plays a critical role in photosynthesis as it allows plants to absorb energy from light. [10] Chlorophyll's green pigmentation may be attributed to its strong blue and red wavelength absorption. Therefore, an indicator of a healthy plant is it appears green in color. Between the red and near infrared wavelengths most plants experience a sharp reflectance rise. This high reflectance value is attributed to interactions within the internal cellular structure of leaves. A plants near infrared reflectance value varies depending on species type, plant stress and canopy state. With seasonal changes, such as autumn, near infrared red reflectance decreases and red reflectiveness increases, resulting in brown, red and yellow leaf colors. Figure 5 depicts spectral signatures for healthy and unhealthy vegetation sample, here one may observe the characteristic discussed above.

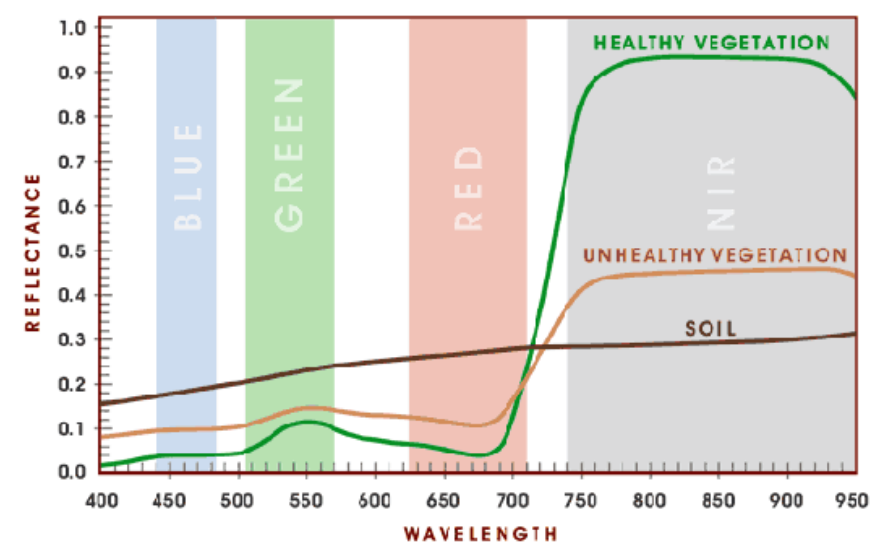


Figure 6: Vegetation reflectance. [11]

\subsubsection{Skin Spectra}

Skin spectrum varies upon several factors (i.e. age, gender, ethnicity, race, etc.). The skin contains different layers, such as the stratum corneum, ${ }^{1}$ the epidermis, the dermis and the hypodermis. The stratum corneum is approximately 10 to $20 \mu \mathrm{m}$ in depth. The epidermis is approximately $50 \mu \mathrm{m}$ on the eyelid and $1500 \mu \mathrm{m}$ on the palms and soles. The absorption coefficient of the epidermis is mainly dependent on the melanin absorption due to the melanosomes ${ }^{2}$ in the epidermis. The dermis is approximately $300 \mu \mathrm{m}$ on the eyelid and $3000 \mu \mathrm{m}$ on the back. The absorption coefficient of the dermis is mainly dependent on the hemoglobin ${ }^{3}$ absorption due to the skin blood flow. Anything beyond these layers is known as the hypodermis. Therefore, the overall skin absorption may be defined by melanin in the visible spectrum and hemoglobin in the NIR, which are proportional to the percentage of melanosomes and whole blood. Each of these layers attribute to the overall reflectance and absorption properties of skin when exposed to incident light are responsible for the spectral response, this behavior may be observed in Figure 7. [16 - 19]

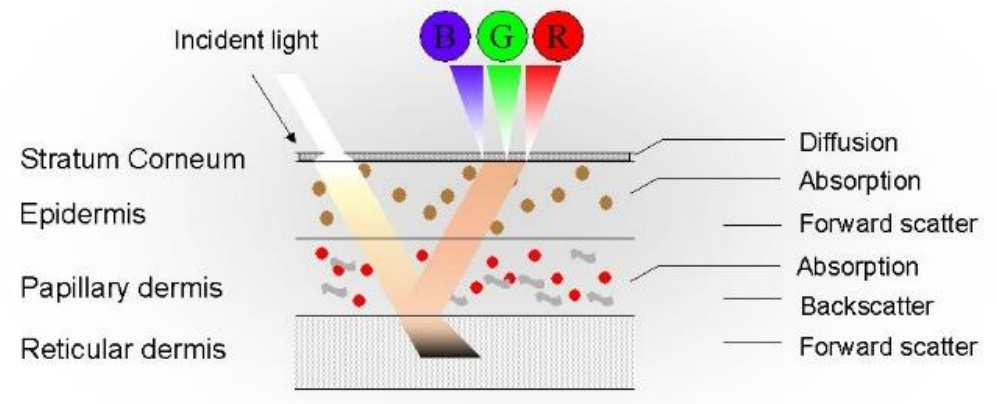

Figure 7: The reflectance and absorption of the skin attributes to the skin coloration of a person. [20]

\footnotetext{
${ }^{1}$ Stratum corneum: the top layer of the epidermis, typically composed of ski oils and skin cells called corneocytes. Corneocytes are regularly replaced through skin peeling and replaced with lower epidermal layers. [12]

${ }^{2}$ Melanosome: an organelle and cellular site of synthesis, storage and transport of melanin. [13] Melanin is the pigment that gives human skin, hair, and eyes their color. Melanin is produced by melanocyte cells. Dark-skinned people contain more melanin than light-skinned people. Exposure to the sun causes melanocytes to increase the production of melanin. [14]

${ }^{3}$ Hemoglobin: iron-containing protein in the red blood cells that transports oxygen to the tissues and returns carbon dioxide from the tissues back to the lungs. [15]
} 
A person skin complexion is based on melanin. There are two major types of melanin: eumelanin and pheomelanin. Eumelanin corresponds to a black or brown pigment while pheomelanin corresponds to red or yellow pigment. For this reason the more eumelanin a person contains the darker their skin complexion will be. [21] Figure 8(a) shows how a person's skin complexion correlates to the percentage of melanosomes.

(a)

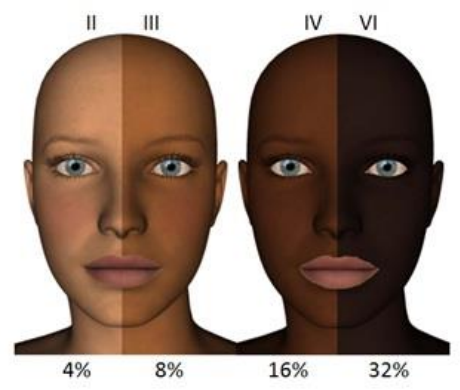

(b)

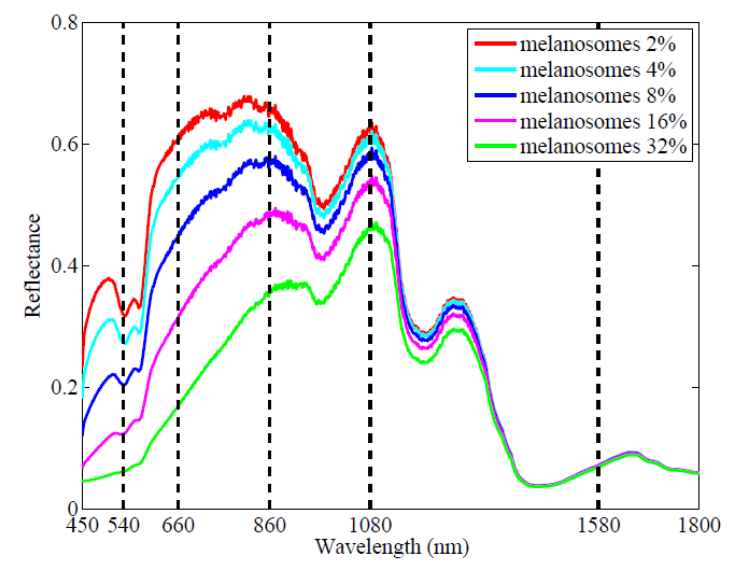

Figure 8: (a) Skin complexion based on the melanosomes percentage. (b) Spectral reflectance of human skin at VIS-SWIR wavelengths [22]

Although these two factors affect the magnitude and shape of the reflectance, some similarities may be observed in behavior to where one may clearly distinguish skin over any other material. For the signatures in Figure 8(b), skin with less melanin appears brighter because it has higher reflectance. Human skin spectral signature exhibits numerous distinctive features (e.g. absorption features) in the visible and NIR regions of the electromagnetic spectrum that can be exploited for skin detection over cluttered backgrounds.[23-25] For instance, human skin is more red than green which can be used to discriminate skin from green vegetation (more green than red). Note also the absorption feature around $980 \mathrm{~nm}$ and the general behavior of skin reflectance above $1000 \mathrm{~nm}$. The normalized difference indices described later take advantage of these features. One may observe that after the SWIR wavelength all percentages of melanosomes behave in a similar manner as they all approach the same curve. This is mainly attributed to the thermal 
emissivity of an individual; essentially all living beings will emit thermal energy as the average temperature of a healthy individual is approximately $37^{\circ} \mathrm{C}$. In addition, some absorption bands of the atmosphere's transmittance may be observed in the NIR and SWIR.

\section{4 Previous Research}

Over the past couple of years, color techniques and hyperspectral band selection [22] have been utilized for skin detection. When detecting, localizing and tracking skin a common question arises, what are the advantages and disadvantages of using RGB images over hyperspectral images and vice versa?

Most systems utilize either pixel-based or region-based methods. Pixel-based methods classify each pixel as skin or non-skin, independently from its neighbors. Region-based methods on the other hand classify a pixel as skin or non-skin based on the spatial arrangements of its findings. Skin modelling is then divided into two subsets, nonparametric or parametric skin distribution modelling algorithms. Nonparametric modelling algorithms estimate skin color distribution from training data set without deriving an explicit model (i.e. normalized lookup table (LUT), Bayes classifier, and self-organized map (SOM), etc.). Parametric modelling algorithms are fast in training and usage and they are theoretically independent to shape of skin distribution from data set (i.e. single Gaussian, Gaussian mixture model, 3D Gaussian in the $\mathrm{YCbCr}$ space, Elliptic boundary model, etc.). All of these methods clustering techniques that were applied to color images may also be used in spectral images. [26]

\subsubsection{Skin Detection using Color Imaging}

Many algorithms are described in the literature for skin detection in color imagery, see [1] for a review. Skin detection approaches using color imagery are based on features derived from different color space representations (e.g. RGB, normalized RGB, YCrCb). The main advantage to these methods is that they are simple and easy to implement based on the desired classification rules. This may become a little more challenging when finding the best color space to work with 
and the corresponding set of rules. Machine learning algorithms have been proposed to find a suitable color space and a set of decision making rules to find the discrimination rules. [26]

Nonparametric methods are fast in training and classification but require large storage space and training datasets. Parametric methods on the other hand may also be fast and require small amounts of storage space. Parametric methods tend to be slower in training and work and their performance is highly dictated by the skin distribution shape. [26]

Some of the obstacles or limitations that one may face when utilizing these techniques are the high occurrence of false alarms. Evidently false alarms are inevitable in most real world scenarios since any object that appears to have a skin color will be classified as skin. In addition most existing algorithms accuracy varies depending on the lighting conditions and the melanosomes percentage on the individual.

\subsubsection{Skin Detection using Hyperspectral Imaging}

Skin detection in hyperspectral imaging has been a recent growing field in security and defense. In Germany, at the University of Koblenz-Landau, students utilized rescue robots with hyperspectral imaging to locate victims after a natural disaster (i.e. earthquake). Their findings showed that skin can be detected using hyperspectral imaging even after a body is covered by debris (i.e. ash). Also, their results indicated that hyperspectral imaging analysis provided a much higher skin detection accuracy rates than detection using color and thermal imaging. [27] Similarly, The University of Eastern Finland utilized hyperspectral imaging data for monitoring and evaluation of skin pigmentations. They were able to estimate the volume fraction of melanosomes, volume fraction of blood, hemoglobin oxygen saturation, along with other skin parameters whose results were later compared to those found in the literature. [28] The Air Force Institute of Technology utilizes existing normalized difference indices in order to detect skin. Normalized difference indices that take advantage of skin spectral features were proposed in order

to detect skin. Their proposed approach significantly reduced the number of false alarms attributed to human-like objects. However, their proposed Normalized Difference Skin Index (NDSI) utilizes 
wavelengths $1580 \mathrm{~nm}$ and $1080 \mathrm{~nm}$, both of which are located in the SWIR. Although this method reduced the number of false alarms the cost for SWIR sensors is rather high. [24,29] These are just some examples of how utilizing hyperspectral images for skin detection has resulted in higher accuracy in comparison to color modeling, as the spectral information allows them to remove objects that appear as skin and even able to clean out signatures on thinks that do not visibly appear as skin. 


\section{Chapter 3: Methods}

In this research hyperspectral image are collected and processed in efforts to find good features for skin detection. The overall goal is to develop features that will help discriminate between skin and non-skin objects in a hyperspectral image. In order to do this we examine collected spectral images and study different normalized difference ratios as feature candidates. The multispectral ratios are calculated for all images and combinations of the ratios are stored into an image cube format to later be processed using the Hyperspectral Image Analysis Toolbox (HIAT). [30] In HIAT, testing and training sets are visually identified. After the testing and training sets are created, the Euclidean distance classifier is applied to the image. Testing and training data sets are used to compute a 2 by 2 confusion matrix. The resulting accuracies are compared for different combinations of features. The flowchart in Figure 9 displays an overview of the methodology.

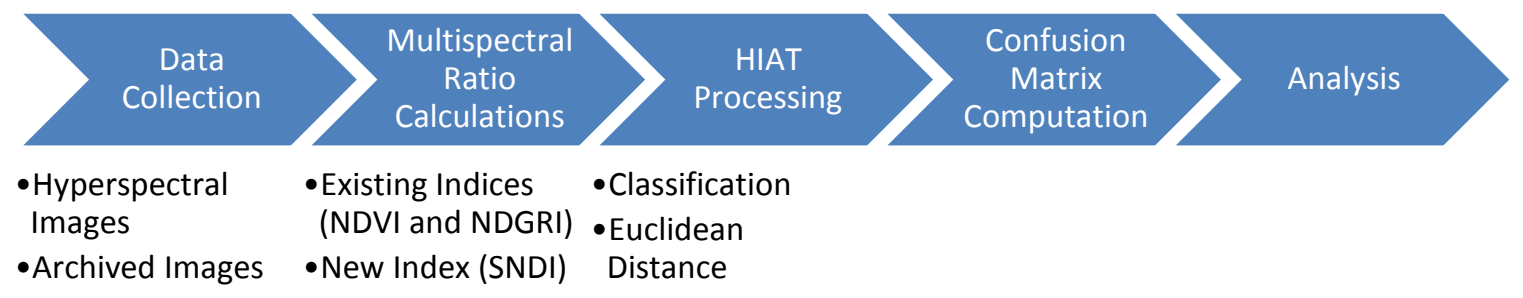

Figure 9: Flow chart methodology.

Some of the hyperspectral images utilized in this research were collected using a Surface Optics' SOC710-VP Hyperspectral Imager. The specifications of this imager are given in Table 2. Notice that the spectral coverage of the imager is between $400-100 \mathrm{~nm}$. This was an important restriction as it is of interest in our work to perform detection using imagers in that spectral range where systems are of significantly lower cost than systems in the NIR where skin detection is easier to detect. Archived data collected with imagers of similar characteristics from collaborators was also used. 
Table 2: SOC710-VP Specifications.

\begin{tabular}{|l|l|}
\hline Spectral Coverage: & $400-1000$ nanometers \\
\hline Spectral Resolution: & 4.6875 nanometer \\
\hline Bands: & 128 \\
\hline Dynamic Range: & 12 -bit \\
\hline Pixels per line: & 696 \\
\hline Speed: & 30 spatial lines per second, 23.2 seconds/cube $(696$ by 520 cube) \\
\hline Focal Length: & Configurable (based on lens used $)$ \\
\hline Lens Type: & C-Mount \\
\hline Weight: & $2.95 \mathrm{Kg}(6.5 \mathrm{lbs})$. \\
\hline Dimensions $(\mathbf{H W L}):$ & $9.5 \times 16.8 \times 22 \mathrm{~cm}(3.75 \times 6.62 \times 8.66$ in. $)$ \\
\hline Power: & 12 -VDC $/ 100-240 \mathrm{VAC}(50-60 \mathrm{~Hz})$ \\
\hline
\end{tabular}

\subsection{Multispectral Ratios}

Band ratios and normalized indices are commonly used in multi/hyperspectral image analysis [31]. Our ultimate goal is to develop a system that can detect human skin over different backgrounds that can be used as part of a search and rescue system, or in defense and security applications (e.g. sniper and dismount detections). Normalized indices are simple to compute and can be used as part of a pre-processing stage to detect regions of interest and reduce the dimensionality of the search space. Normalized difference indices are often used for detection as they are invariant to offset and scaling that occur due to transitivity and scattering. We look at combinations that include indices that highlight background (e.g. vegetation) and those that highlight skin.

A common normalized index used in remote sensing is the Normalized Difference Vegetation Index (NDVI) [31]

$$
N D V I=\frac{\rho_{N I R}-\rho_{\text {red }}}{\rho_{N I R}+\rho_{\text {red }}}=\frac{\rho_{860}-\rho_{650}}{\rho_{860}+\rho_{650}} .
$$


NDVI has been used in remote sensing to assess whether the target being observed contains live green vegetation or not. It takes advantage of the red edge feature present in vegetation spectra. The reader is referenced to (Schowengerdt, 2007) for further details.

Normalized difference indices have been proposed as features for skin detection. In (Nunez et al., 2008; Nunez, 2015), the Normalized Difference Skin Index (NDGRI) and the Normalized Difference Green-Red Index (NDGRI) were proposed as computationally simple features that can provide high probability of detection with low probability of false alarm.

The NDSI is a function of reflectance at $1080 \mathrm{~nm}$ and $1580 \mathrm{~nm}$. The reflectance at $1080 \mathrm{~nm}$ is the location of a local maximum (see Figure $8(\mathrm{~b})$ ) of the reflectance of skin in the NIR where melanosome absorption dominates. A stable yet low-valued reflectance feature in skin spectra is chosen at $1580 \mathrm{~nm}$, in order to ensure a large difference between melanin-dominated and waterdominated portions of the skin spectra. The NDSI is given by [23, 24, 29],

$$
N D S I=\frac{\rho_{1080}-\rho_{1580}}{\rho_{1080}+\rho_{1580}} .
$$

The color green comprises of the $495 \mathrm{~nm}$ through $570 \mathrm{~nm}$ region of the spectra while green color ranges from $620 \mathrm{~nm}$ through $750 \mathrm{~nm}$. As Figure 1 shows, human skin is more red than green while vegetation is more green than red. The NDGRI is given by [23, 24, 29],

$$
N D G R I=\frac{\rho_{\text {green }}-\rho_{\text {red }}}{\rho_{\text {green }}+\rho_{\text {red }}}=\frac{\rho_{510}-\rho_{650}}{\rho_{510}+\rho_{650}} \text {. }
$$

Human skin will result in negative values of NDGRI while vegetation will result in positive value. Clearly these differences can be used to discriminate between skin over a vegetation background. In equations $(1)-(3), \rho_{\mathrm{NIR}}, \rho_{\mathrm{red}}$, and $\rho_{\text {green }}$ are the reflectance values of near-infrared, red, and green. In our experiments, the NIR wavelength value was set at $860 \mathrm{~nm}$, the red at 650 $\mathrm{nm}$, and the green at $510 \mathrm{~nm}$. The results of [23, 24, 29] were very encouraging but the requirements of a hyperspectral imager covering the VIS and NIR ranges (400nm to $1700 \mathrm{~nm}$ ) presents a cost challenge for implementation. 
In this work, we limited our focus to imager in the $400 \mathrm{~nm}$ to $1000 \mathrm{~nm}$. Cost of imagers in this spectral range is 4 to 5 times lower than those covering up to $1800 \mathrm{~nm}$. Hence we will not use the NDSI in (1), An interesting spectral feature not explored in $[23,24,29]$ is the spectral difference between the skin reflectance peak around $800 \mathrm{~nm}$ and the absorption feature around 980 nm. Based on this feature, we propose the Skin Normalized Difference Index (SNDI)

$$
S N D I=\frac{\rho_{980}-\rho_{800}}{\rho_{980}+\rho_{800}} .
$$

A weakness of this feature compared to the NDSI is that and the percentage of melanosomes increase the difference becomes smaller. The proposed ratio SNDI was found by examining the skin spectral signatures shown in Figure 8(b) to find a discriminating factor. A significant reduction in the reflectance may be observed between $800 \mathrm{~nm}$ and $980 \mathrm{~nm}$ because of the absorption features at $980 \mathrm{~nm}$; for this reason these values were selected for the proposed ratio.

\subsection{Feature Combinations}

After all the multispectral ratios are computed six feature cubes are created by combining multiple ratio combinations. Table 3 shows the different feature combinations cubes analyzed using HIAT.

Table 3: Feature Combinations studied in the experiments.

\begin{tabular}{r|l} 
Image Label & Feature Combinations \\
\hline One & All Bands \\
Two & NDGRI+NDVI+SNDI \\
Three & NDGRI+NDVI \\
Four & NDGRI+SNDI \\
Five & NDVI+SNDI \\
Six & NDGRI \\
Seven & SNDI
\end{tabular}

The pairings for each subset cube was made out of all possible ratio combinations. We did not present results for NDVI alone as it is strictly a vegetation index ratio that is not helpful in skin detection by itself but we think it may improve discrimination when combined with other indices. 


\subsection{Hyperspectral Image Analysis Toolbox (HIAT)}

The HIAT is a MATLAB toolbox created at the University of Puerto Rico - Mayaguez (UPRM). It is a computational environment that contains hyperspectral image processing algorithms developed at UPRM Laboratory for Applied Remote Sensing and Image Processing (LARSIP). Some of the features this toolbox contains are image enhancement, band subset section, unmixing, feature extraction, supervised classification, and unsupervised classification. Many of these features can be applied to multi-feature imaged organized in a cube format not only to hyperspectral imagery.

\subsection{Classification Analysis}

To evaluate how good different individual or combinations of features were in skin discrimination, we perform a simple Euclidean distance classification on the feature image and evaluated the performance of the classifier between the skin and non-skin class. HIAT was utilized to conduct the classification analysis. Testing and training sets were created for each image through visual examination. The supervised training and testing sets were created by selecting areas using polygons in the images and labeling them with the corresponding class as shown in Figure 10. The same testing and training polygons and labels were utilized for all of the feature combinations for a given image. After the testing and training sets were created, the Euclidean distance classifier was applied to all feature cubes. Classification accuracies were used as proxies for discrimination power of the selected features.

However, supervised training data is driven by the desired map themes and not by characteristics of the data itself there is no assurance that the classes will be distinguishable from one another. After the testing and training sets were created, the Euclidean distance classifier was applied to the image; this classifier is available in HIAT. 


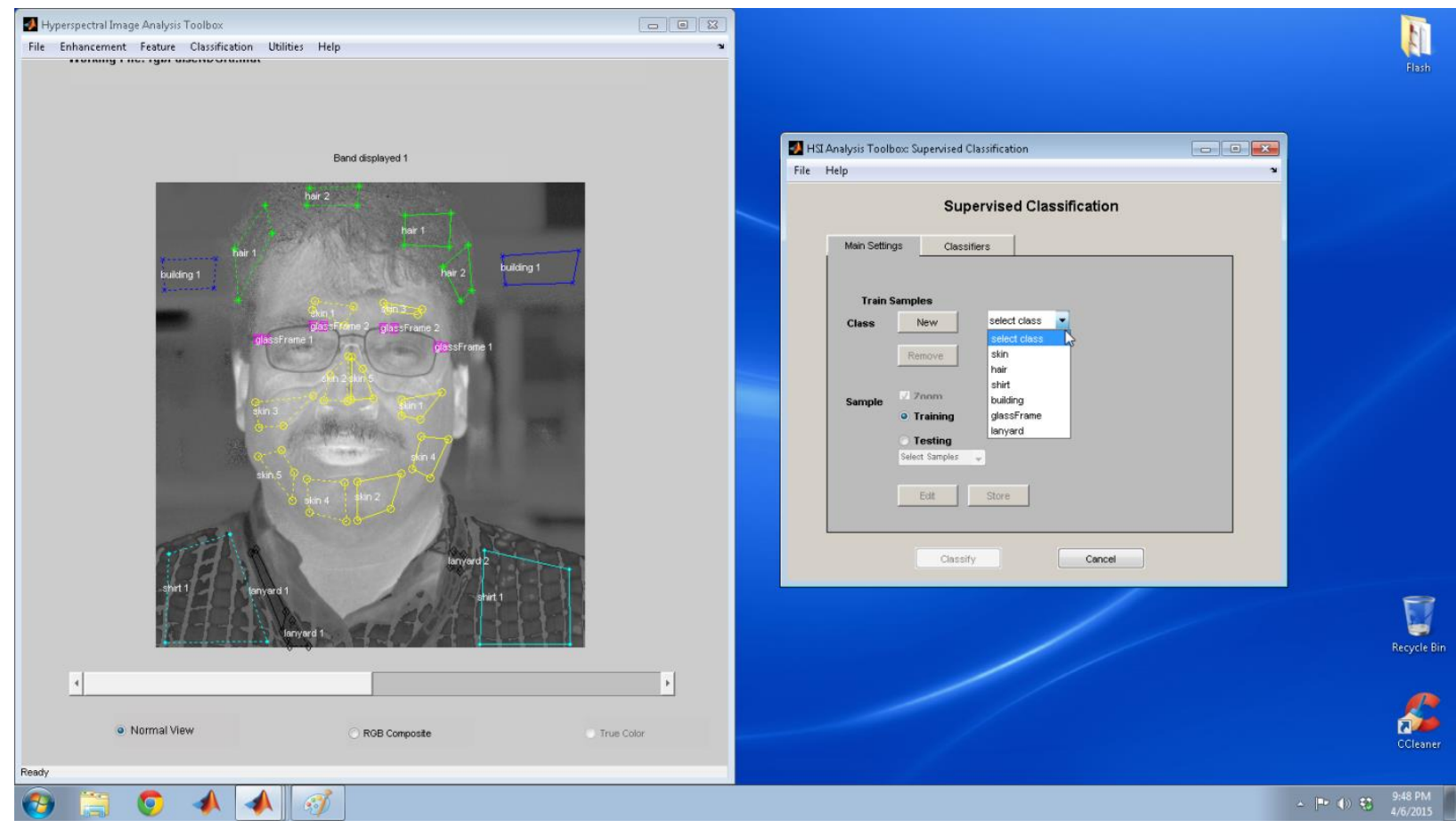

Figure 10: Subject one's testing and training polygons.

\subsubsection{Euclidean Distance Classifier}

In the experiments, the Euclidean distance classifier was used as the supervised classifier. The Euclidean distance classifier is a simple classification rule where the pixel under evaluation is assigned to the class whose mean is the closest to the sample under evaluation.

In order to compute the minimum distance classifier all training vectors are separated by class. The mean for each class, $\mu_{\mathrm{k}}$ where $\mathrm{k}=1, \ldots \mathrm{m}$ are then computed. The closest mean to a test vector $\mathrm{x}$ is computed using Euclidean distance. Based on this result the corresponding class is predicted for the vector. [31]

\subsubsection{Confusion matrices}

Confusion matrices, also known as contingency tables in statistics, are often used as an accuracy assessment tool of the classification result. This one is created utilizing the reference (user) data and the classified (producer) data. The reference refers to the number of times an event occurred, while the producer refers to the number of times an event was forecast. A hit refers to the instances where an event occurrence was forecasted. A false alarm refers to the instances where 
an event was forecasted but did not occur. A miss refers to the instances where an event did not occur but was forecasted. A null event refers to the instances where an event did not occur and was not forecast. The resulting confusion matrix is a $2 \times 2$ matrix shown in Table 4. [32]

Table 4: Contingency Table.

\begin{tabular}{cc|c|c|} 
& & \multicolumn{3}{c}{ event occurred? } \\
\multirow{2}{*}{ event } & yes & yes & ho \\
forecast? & & $\mathrm{h}$ & false alarm \\
\cline { 2 - 4 } & no & miss & f null event \\
& & $\mathrm{m}$ & $\mathrm{q}$ \\
\hline
\end{tabular}

The accuracy of the Euclidean distance classification results are summarized in confusion matrices for training and testing data which is automatically provided by HIAT. These matrices are further simplified into contingency tables such as Table 4 for skin and non-skin classes. For any given $\mathrm{N}$ by $\mathrm{M}$ confusion matrix, the first value is declared as the hits (shown in blue in Table 5), which mark the number of pixels that were classified as skin and were skin. The false alarms are the addition of all pixels that were classified as skin but were not skin (shown in red in Table 5). The misses are the addition of all pixels that were not classified as skin and were skin (shown in green in Table 5). The null events are the addition of all pixels that were not classified as skin and were not skin (shown in orange in Table 5).

Table 5: $\mathrm{N}$ by $\mathrm{M}$ matrix transformation.

\begin{tabular}{|c|c|c|c|c|}
\hline 1,1 & 1,2 & 1,3 & $\rightarrow$ & $1, \mathrm{M}$ \\
\hline 2,1 & 2,2 & 2,3 & $\rightarrow$ & $2, \mathrm{M}$ \\
\hline 3,1 & 3,2 & 3,3 & $\rightarrow$ & $3, \mathrm{M}$ \\
\hline$\downarrow$ & $\downarrow$ & $\downarrow$ & $\searrow$ & $\downarrow$ \\
\hline $\mathrm{N}, 1$ & $\mathrm{~N}, 2$ & $\mathrm{~N}, 3$ & $\rightarrow$ & $\mathrm{N}, \mathrm{M}$ \\
\hline
\end{tabular}


After computing these four values for all training and testing matrices the $\mathrm{N}$ by $\mathrm{M}$ matrix is simplified into a two by two contingency table as shown Table 6 .

Table 6: Simplified Contingency Table and Accuracy Assessment.

\begin{tabular}{|c|c|c|c|c|}
\hline & \multicolumn{2}{|c|}{$\begin{array}{c}\text { Field Verified Density } \\
\text { Class }\end{array}$} & \multirow[b]{2}{*}{ User's Accuracy } \\
\hline & & Skin & Non-Skin & \\
\hline \multirow{3}{*}{$\begin{array}{c}\text { Interpreted } \\
\text { Density Class }\end{array}$} & Skin & $\begin{array}{c}\mathrm{h}(1,1) \\
\mathrm{I}\end{array}$ & $\begin{array}{c}\mathrm{f}(1,2) \\
\text { II }\end{array}$ & $\begin{array}{l}\text { Positive Predicted Value } \\
\qquad \frac{I}{I+I I}\end{array}$ \\
\hline & Non-Skin & $\begin{array}{c}\mathrm{m}(2,1) \\
\mathrm{III}\end{array}$ & $\begin{array}{l}\text { n }(2,2) \\
\text { IV }\end{array}$ & $\begin{array}{l}\text { Negative Predicted Value } \\
\qquad \frac{I V}{I I I+I V}\end{array}$ \\
\hline & $\begin{array}{l}\text { Producer's } \\
\text { Accuracy }\end{array}$ & $\begin{array}{l}\text { Sensitivity } \\
\frac{I}{I+I I I}\end{array}$ & $\begin{array}{l}\text { Specificity } \\
\frac{I V}{I I+I V}\end{array}$ & $\begin{array}{l}\text { Total Accuracy } \\
\frac{I+I V}{I+I I+I I I+I V}\end{array}$ \\
\hline
\end{tabular}

\subsubsection{Accuracy Assessment}

The accuracy assessment quantifies how well or how poor something was done by the classifiers. This assessment reflects the difference between the classification outcomes and the reference data. Therefore, if the reference data is inaccurate the assessment might indicate a poor classification when in all reality it is good. After an image is collected and the confusion matrix is created the user, producer, and total accuracy percentages are calculated. Table 6 shows how these values are computed from the simplified contingency table. The producer's accuracy describes the probability that the classifier has labeled an image pixel into Class A given that the ground truth is Class A, and contains the sensitivity and specificity values; while the user's accuracy describes the probability that a pixel is Class A given that the classifier has labeled the pixel into Class A, and contains the positive and negative predicted values. [32, 33]

From the sensitivity and specificity classifications one may obtain the false positive, false negative rate, and power, which are all error types. The equations for these error types are shown in Table 7. The false positive rate $(\alpha)$ is known as an error of the first kind, is the probability of 
rejecting the null hypothesis given that it is true. The false negative rate $(\beta)$ is known as an error of the second kind, is the probability of accepting the null hypothesis given that it is false.

Table 7: Error Types.

\begin{tabular}{|c|c|}
\hline Calculation & Equation \\
\hline $\begin{array}{c}\text { False positive rate }(\alpha) \\
\bullet \quad \text { type I error (false alarm) }\end{array}$ & $=1-$ specificity \\
\hline $\begin{array}{c}\text { False negative rate }(\beta) \\
\bullet \quad \text { type II error (miss) }\end{array}$ & $=1-$ sensitivity \\
\hline
\end{tabular}

\subsection{Hyperspectral Images used in the Experiments}

Four distinct subjects were utilized in this experiment, an RGB composite of each of the hyperspectral image collected for each subjects may be observed in Figure 1l. Although the exact instrumentation used to collect subject one, two, and three's images the bandwidth and pixel size was retrieved from the data provided. These three cubes are made up of 160 close contiguous bands that ranged from $400 \mathrm{~nm}$ to $1000 \mathrm{~nm}$. The size of the image for subject-one is 1480 pixels by 1600 pixels, subject-two's image is 1056 pixels by 1600, and subject-three's image is 914 pixels by 1600. Subject four's image was collected with the SOC710-VP hyperspectral imager, whose specifications are listed in Table 2. The first three subjects were placed in front of a building and in return collected a face image. The fourth subject was placed in a vegetation background. After all images had been collected, the feature combinations were then computed for each individual's image. Testing and training polygons where then used for images classification. These sets were then used to compute the Euclidean distance classifier in HIAT. The resulting testing and training confusion matrices from the classifier were then simplified into contingency tables in MATLAB. The contingency tables were then utilized to assess the accuracy for each of the feature combinations. 


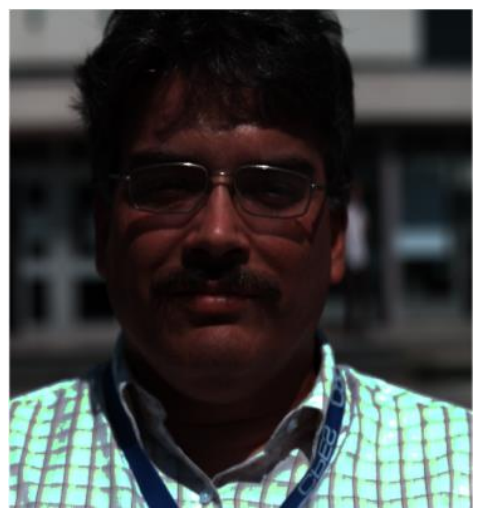

(a)

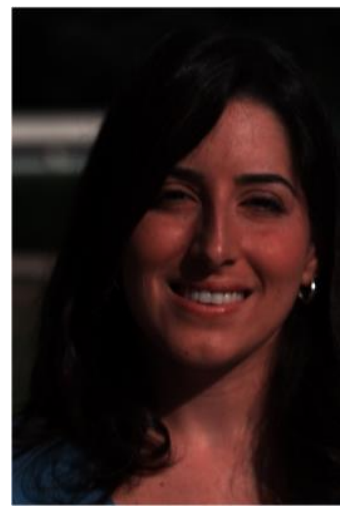

(b)

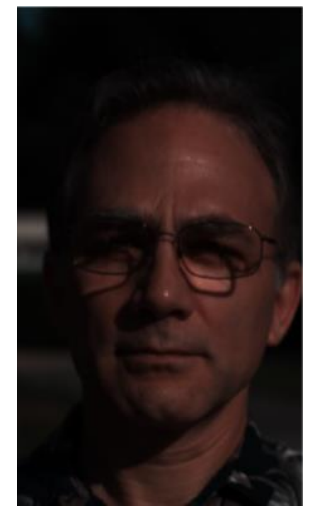

(c)

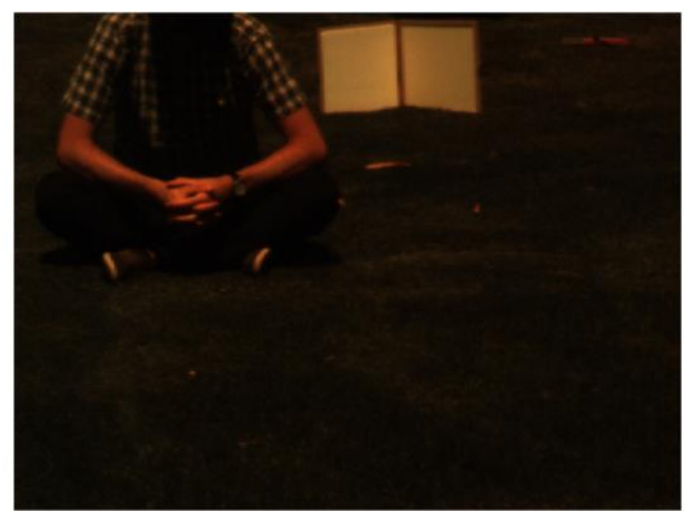

(d)

Figure 11: True color RGB composites using R $\sim 50 \mathrm{~nm}, \mathrm{G} \sim 510 \mathrm{~nm}$ and $\mathrm{B} \sim 475 \mathrm{~nm}$ for each subject. (a) Subject-One. (b) Subject-Two. (c) Subject- Three. (d) Subject-Four. 


\section{Chapter 4: Results and Discussion}

This chapter presents experimental results comparing classification results using all feature combinations for the four subjects. The testing and training polygons used for classification for each of the subjects are shown in Figure 12.

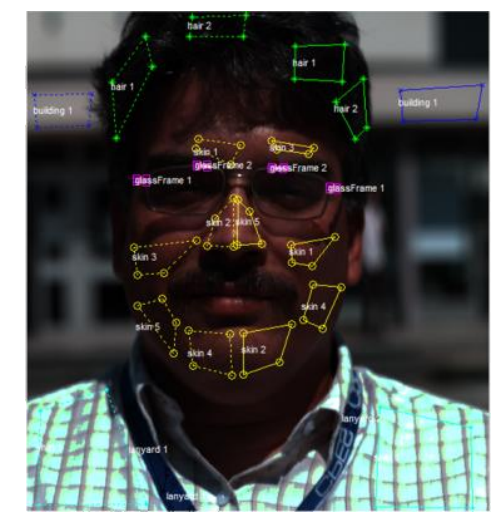

(a)

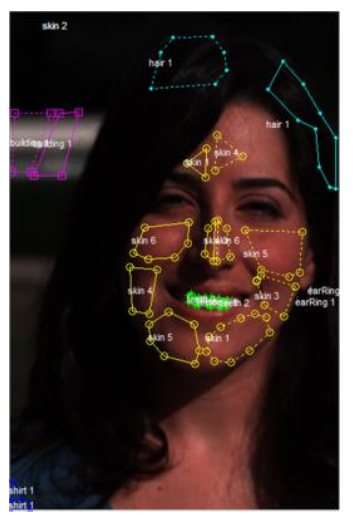

(b)

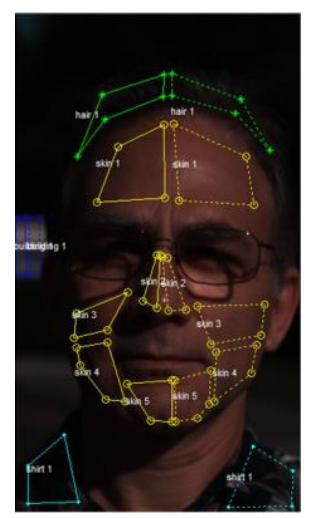

(c)

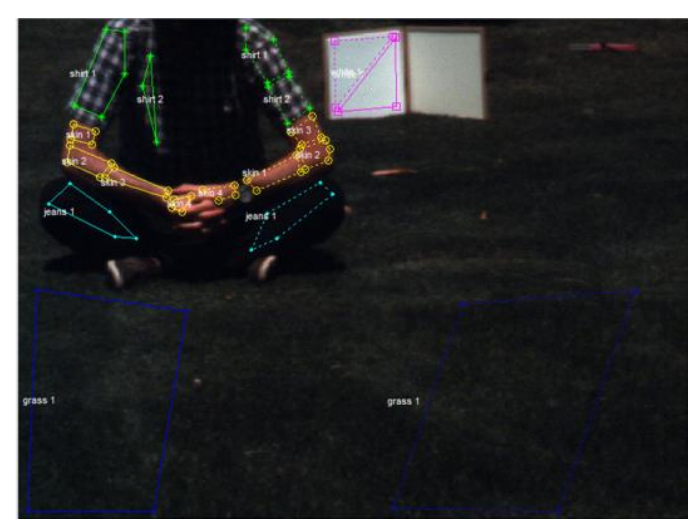

(d)

Figure 12: True color RGB composites with the corresponding testing and training polygons for each subject. (a) Subject-One. (b) Subject-Two. (c) Subject-Three. (d) Subject-Four.

\subsection{Subject-One}

Figure 13 (a) - (c) show the results of a color palette applied to each of the ratio image for the face image of subject-one. Figure 13(d) shows a false RGB composite where NDGRI is in the red band, NDVI is in the green band and SNDI is in the blue band. 


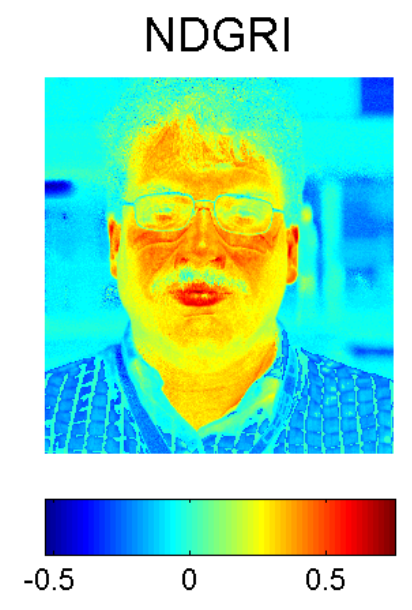

(a)

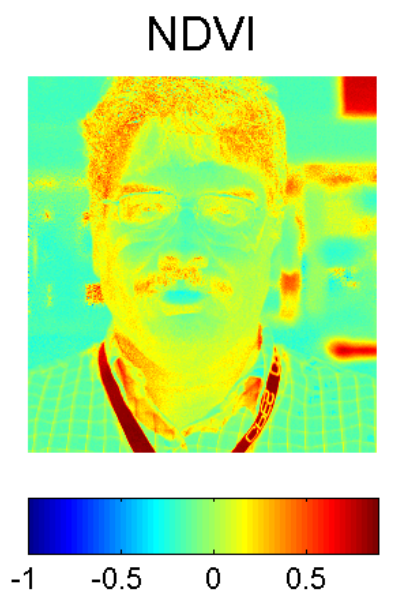

(b)

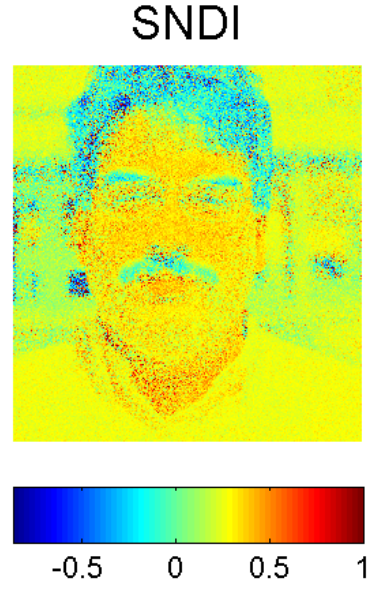

(c)

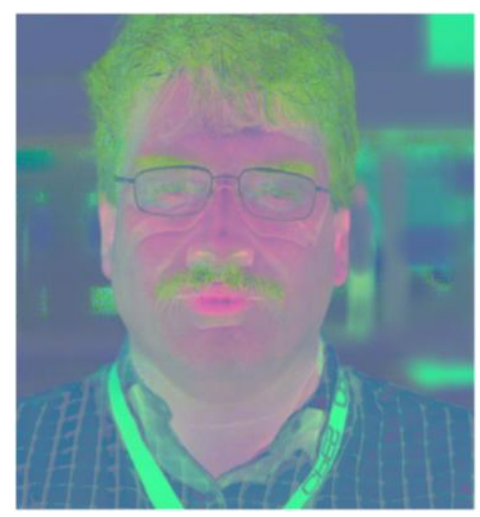

(d)

Figure 13: Color palettes for (a) NDVI, (b) NDGRI, (c) SNDI, and (d) RGB composite using RNDGRI, G-NDVI and B-SNDI.

The face is clearly highlighted in Figure 13(b) and Figure 13(c) for NDGRI and SNDI, respectively. However, the face is not highlighted in Figure 13(a), NDVI; this result is due to the fact that NDVI is designed for detection of vegetation coverage. While subject-one's cube was being collected, a pedestrian's presence was captured in the background near subject-one's left ear. Surprisingly NDGRI detected this person's presence. Visual assessment suggests that NDGRI produces the best discrimination between skin and non-skin. In the RGB composite, the face of subject-one and the skin of the person in the background are shown in varying pink tones. 
Classification accuracy results for all feature combination images in Table 3 using the training and testing samples shown in Figure 12(a) are shown in Figure 14 for subject-one. In the binary images, the white color represents the skin class and the black is the non-skin class. 


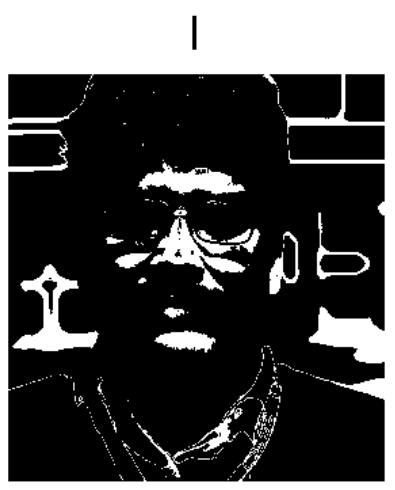

III

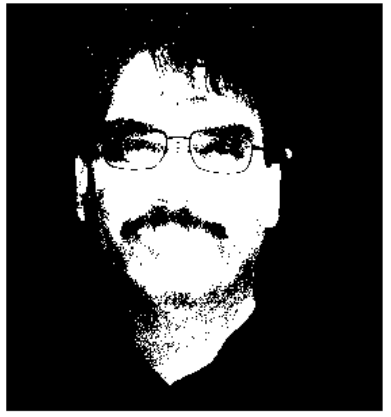

V

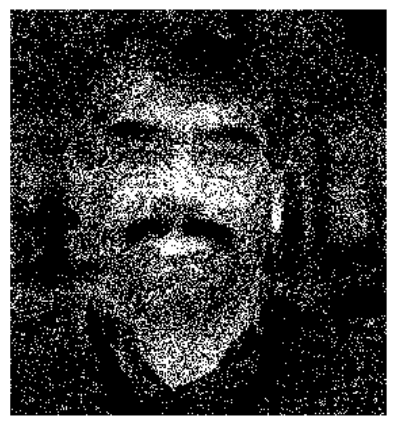

VII

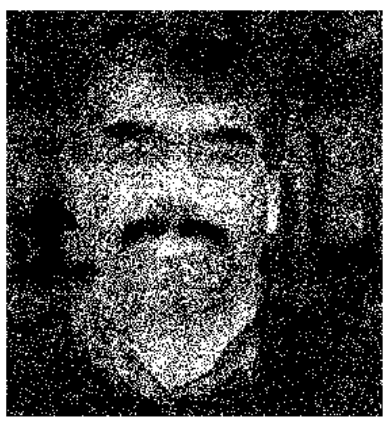

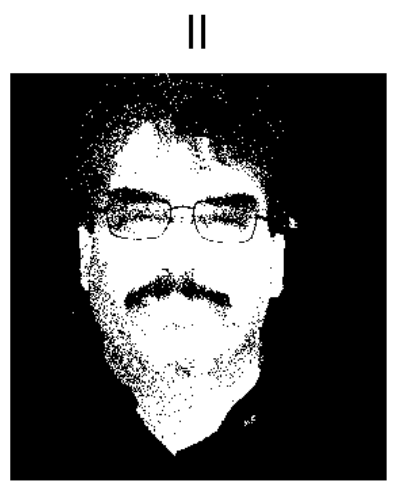

IV

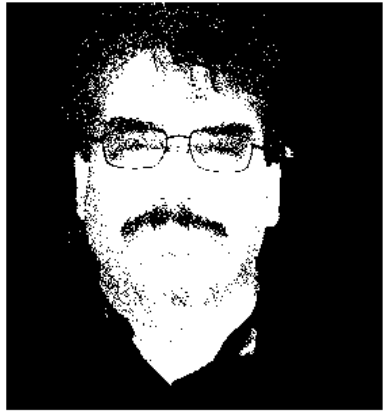

VI

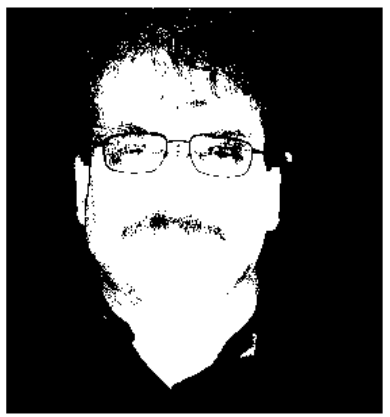

Figure 14: Binary classification of skin and non-skin: I) Full HSI cube.

II) NDGRI+NDVI+SNDI. III) NDGRI+NDVI. IV) NDGRI + SNDI. V) NDVI+SNDI. VI) NDGRI. VII) SNDI. 
Accuracy results for subject-one are shown in Table 8. NDGRI had the highest overall accuracy results, based on accuracy assessment in Table 8 and the error rates in Table 9. For all zero error percentage the cells were highlighted in red and the text color was displayed in red, if a cell has a zero value and does not have these characteristics it simply means that the values are fairly small and cannot be displayed as all values were rounded to the nearest hundredth. When all three indices were combined, we can see in the classification maps that the number of false alarms decreased causing some of the facial features to become much clearer in the binary classifications. In addition, one may observe a person in the background of subject-one image next to his left ear. Although this person's facial features are not clear his presence is clearly visible in NDGRI's palette color composite, the RGB composite, and in multiple binary classifications.

Table 8: Accuracy assessment for subject-one face image.

\begin{tabular}{|c|c|c|c|c|c|c|c|c|c|c|}
\hline & & & & $\begin{array}{c}\text { All } \\
\text { Bands }\end{array}$ & $\begin{array}{c}\text { NDGRI, } \\
\text { NDVI\& } \\
\text { SNDI }\end{array}$ & $\begin{array}{l}\text { NDGRI } \\
\& \text { NDVI }\end{array}$ & $\begin{array}{l}\text { NDGRI } \\
\& \text { SNDI }\end{array}$ & $\begin{array}{c}\text { NDVI \& } \\
\text { SNDI }\end{array}$ & NDGRI & SNDI \\
\hline \multirow{10}{*}{ Subject One } & \multirow{5}{*}{ Test } & \multirow{2}{*}{$\begin{array}{l}\text { non- } \\
\text { skin }\end{array}$} & producer & 78.93 & 98.01 & 92.45 & 99.40 & 83.00 & 99.99 & 86.30 \\
\hline & & & user & 99.00 & 99.43 & 99.96 & 99.33 & 93.51 & 99.81 & 93.37 \\
\hline & & \multirow[t]{2}{*}{ skin } & producer & 90.13 & 98.33 & 99.86 & 98.11 & 71.62 & 99.46 & 75.74 \\
\hline & & & user & 25.62 & 94.33 & 77.01 & 98.32 & 46.10 & 99.97 & 58.27 \\
\hline & & \multicolumn{2}{|c|}{ total } & 79.76 & 98.09 & 93.94 & 99.06 & 81.08 & 99.85 & 84.17 \\
\hline & \multirow{5}{*}{ Train } & \multirow{2}{*}{$\begin{array}{l}\text { non- } \\
\text { skin }\end{array}$} & producer & 99.71 & 100.00 & 100.00 & 100.00 & 99.07 & 100.00 & 98.93 \\
\hline & & & user & 97.57 & 99.91 & 99.99 & 99.90 & 94.11 & 99.99 & 93.21 \\
\hline & & \multirow{2}{*}{ skin } & producer & 67.16 & 98.31 & 99.90 & 98.13 & 42.60 & 99.90 & 38.52 \\
\hline & & & user & 94.53 & 100.00 & 100.00 & 100.00 & 83.16 & 99.99 & 80.88 \\
\hline & & \multicolumn{2}{|c|}{ total } & 97.42 & 99.91 & 99.99 & 99.90 & 93.56 & 99.99 & 92.59 \\
\hline
\end{tabular}

Table 9: Error rates for subject-one face image.

\begin{tabular}{|c|c|c|c|c|c|c|c|c|c|}
\hline & & & $\begin{array}{c}\text { All } \\
\text { Bands }\end{array}$ & $\begin{array}{c}\text { NDGRI, } \\
\text { NDVI\& } \\
\text { SNDI } \\
\end{array}$ & $\begin{array}{l}\text { NDGRI } \\
\& \text { NDVI }\end{array}$ & $\begin{array}{l}\text { NDGRI } \\
\& \text { SNDI }\end{array}$ & $\begin{array}{c}\text { NDVI \& } \\
\text { SNDI } \\
\end{array}$ & NDGRI & SNDI \\
\hline \multirow{4}{*}{ Subject One } & \multirow{2}{*}{ Test } & False Positive Rate & 21.07 & 1.99 & 7.55 & 0.60 & 17.00 & 0.01 & 13.70 \\
\hline & & False Negative Rate & 9.87 & 1.67 & 0.14 & 1.89 & 28.38 & 0.54 & 24.26 \\
\hline & \multirow{2}{*}{ Train } & False Positive Rate & 0.29 & 0.00 & 0.00 & 0.00 & 0.93 & 0.00 & 1.07 \\
\hline & & False Negative Rate & 32.84 & 1.69 & 0.10 & 1.87 & 57.40 & 0.10 & 61.48 \\
\hline
\end{tabular}




\subsection{Subject-Two}

The second image corresponds to subject-two. Subject-two's scene is similar to subjectone's scene. Figure 15 (a) - (c) shows the results of a color palette applied to each of the ratio images and Figure 15(d) shows a false RGB composite for subject-two.

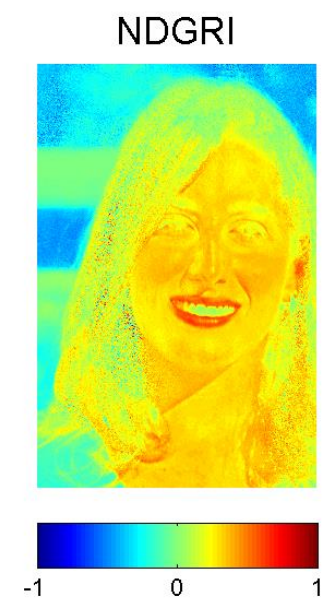

(a)

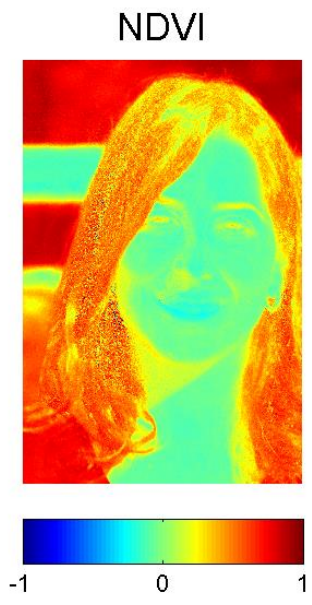

(b)

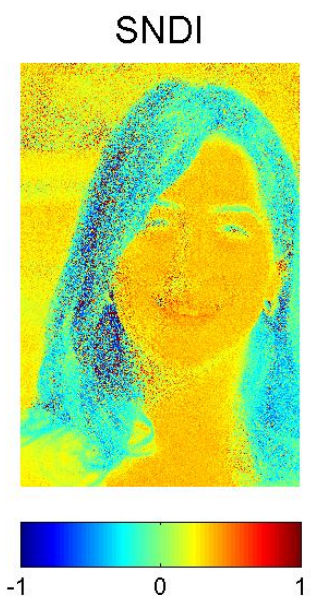

(c)

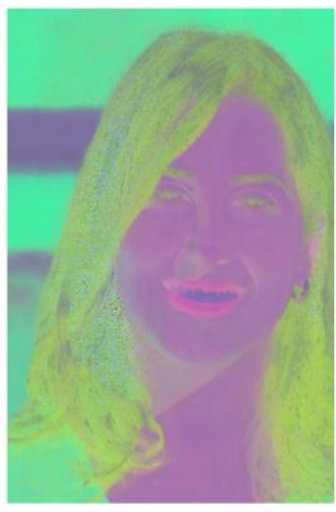

(d)

Figure 15: Color palettes for (a) NDVI, (b) NDGRI, (c) SNDI, and (d) RGB composite using RNDGRI, G-NDVI and B-SNDI.

Classification results for all feature combinations in Table 3 using the training and testing samples shown in Figure 12(b) are shown in Figure 16 for subject-two. In the binary images, the white color represents the skin class and the black is the non-skin class. 

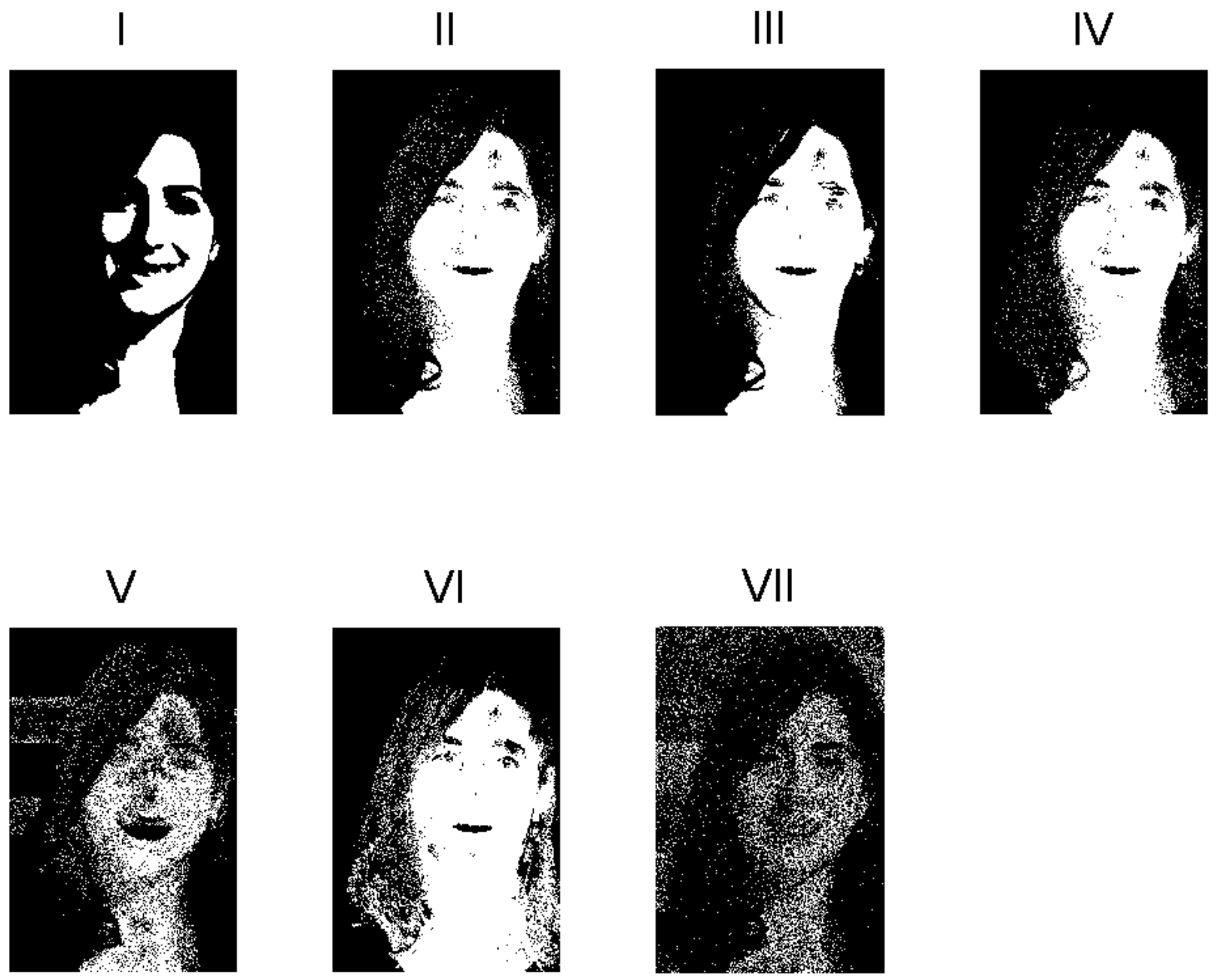

Figure 16: Binary classification of skin and non-skin: I) Full HSI cube.

II) NDGRI+NDVI+SNDI. III) NDGRI+NDVI. IV) NDGRI + SNDI.

V) NDVI+SNDI. VI) NDGRI. VII) SNDI.

Once more, NDGRI had the highest overall accuracy results, based on accuracy assessment in Table 10 and the error rates in Table 11. 
Table 10: Accuracy assessment for subject-two face image.

\begin{tabular}{|c|c|c|c|c|c|c|c|c|c|c|}
\hline & & & & $\begin{array}{c}\text { All } \\
\text { Bands }\end{array}$ & $\begin{array}{c}\text { NDGRI, } \\
\text { NDVI\& } \\
\text { SNDI }\end{array}$ & $\begin{array}{l}\text { NDGRI } \\
\& \text { NDVI }\end{array}$ & $\begin{array}{l}\text { NDGRI } \\
\& \text { SNDI }\end{array}$ & $\begin{array}{c}\text { NDVI \& } \\
\text { SNDI }\end{array}$ & NDGRI & SNDI \\
\hline \multirow{10}{*}{ Subject Two } & \multirow{5}{*}{ Test } & \multirow{2}{*}{$\begin{array}{l}\text { non- } \\
\text { skin }\end{array}$} & producer & 96.31 & 99.86 & 99.86 & 99.68 & 80.30 & 99.67 & 61.55 \\
\hline & & & user & 99.05 & 99.22 & 99.71 & 99.78 & 92.11 & 99.60 & 93.36 \\
\hline & & \multirow[t]{2}{*}{ skin } & producer & 99.15 & 99.32 & 99.75 & 99.81 & 92.13 & 99.65 & 89.49 \\
\hline & & & user & 96.70 & 99.88 & 99.88 & 99.72 & 80.33 & 99.71 & 49.24 \\
\hline & & \multicolumn{2}{|c|}{ total } & 97.79 & 99.57 & 99.80 & 99.75 & 85.81 & 99.66 & 69.77 \\
\hline & \multirow{5}{*}{ Train } & \multirow{2}{*}{$\begin{array}{l}\text { non- } \\
\text { skin }\end{array}$} & producer & 94.43 & 99.98 & 99.99 & 99.98 & 91.14 & 99.99 & 82.50 \\
\hline & & & user & 100.00 & 99.58 & 99.89 & 99.83 & 94.72 & 99.49 & 93.79 \\
\hline & & \multirow[t]{2}{*}{ skin } & producer & 100.00 & 98.82 & 99.67 & 99.52 & 83.04 & 98.56 & 70.97 \\
\hline & & & user & 83.20 & 99.95 & 99.96 & 99.96 & 73.74 & 99.97 & 43.29 \\
\hline & & \multicolumn{2}{|c|}{ total } & 95.64 & 99.68 & 99.91 & 99.86 & 89.27 & 99.61 & 80.67 \\
\hline
\end{tabular}

Table 9: Error rates for subject-two face image.

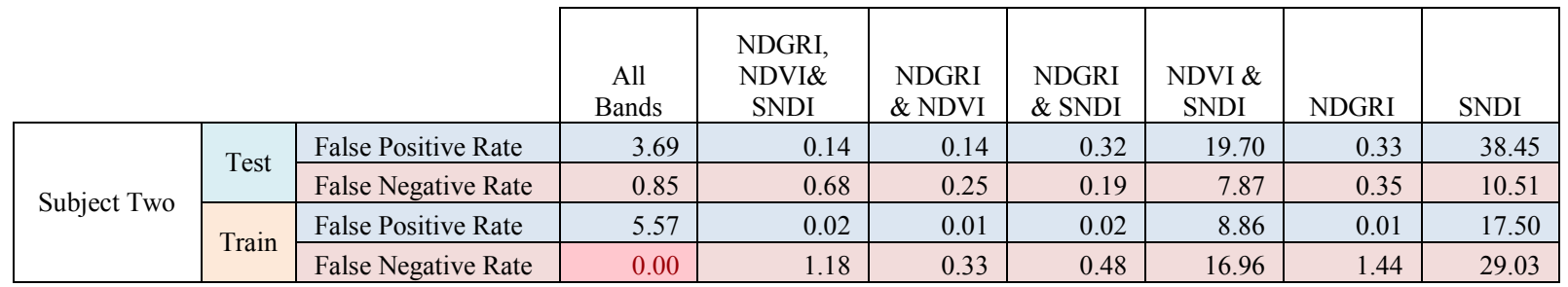

\subsection{Subject-Three}

The third image corresponds to subject-three. Subject-three's scene is similar to subjectone's scene. Figure 17 (a) - (c) shows the results of a color palette applied to each of the ratio images and Figure 17(d) shows a false RGB composite for subject-three. 


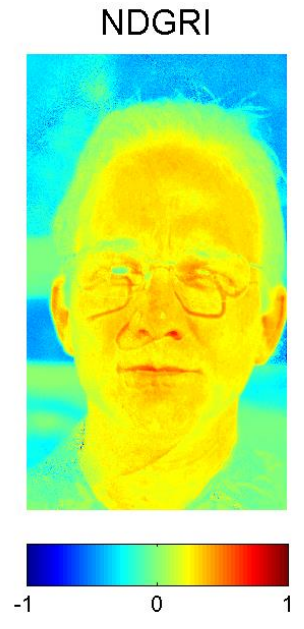

(a)

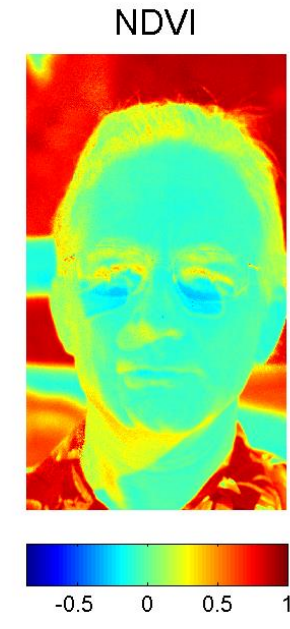

(b)

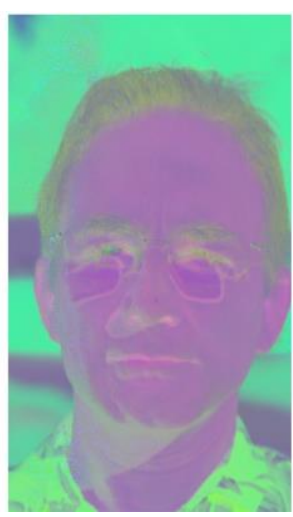

(d)

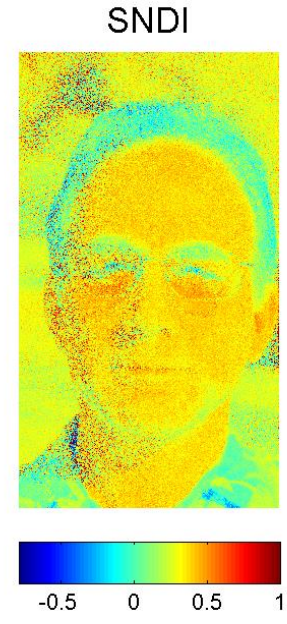

(c)

Figure 17: Color palettes for (a) NDVI, (b) NDGRI, (c) SNDI, and (d) RGB composite using R-
NDGRI, G-NDVI and B-SNDI. Classification results for all feature combinations in Table 3 using the training and testing samples shown in Figure 12(c) are shown in Figure 18 for subject-three. In the binary images, the white color represents the skin class and the black is the non-skin class. 

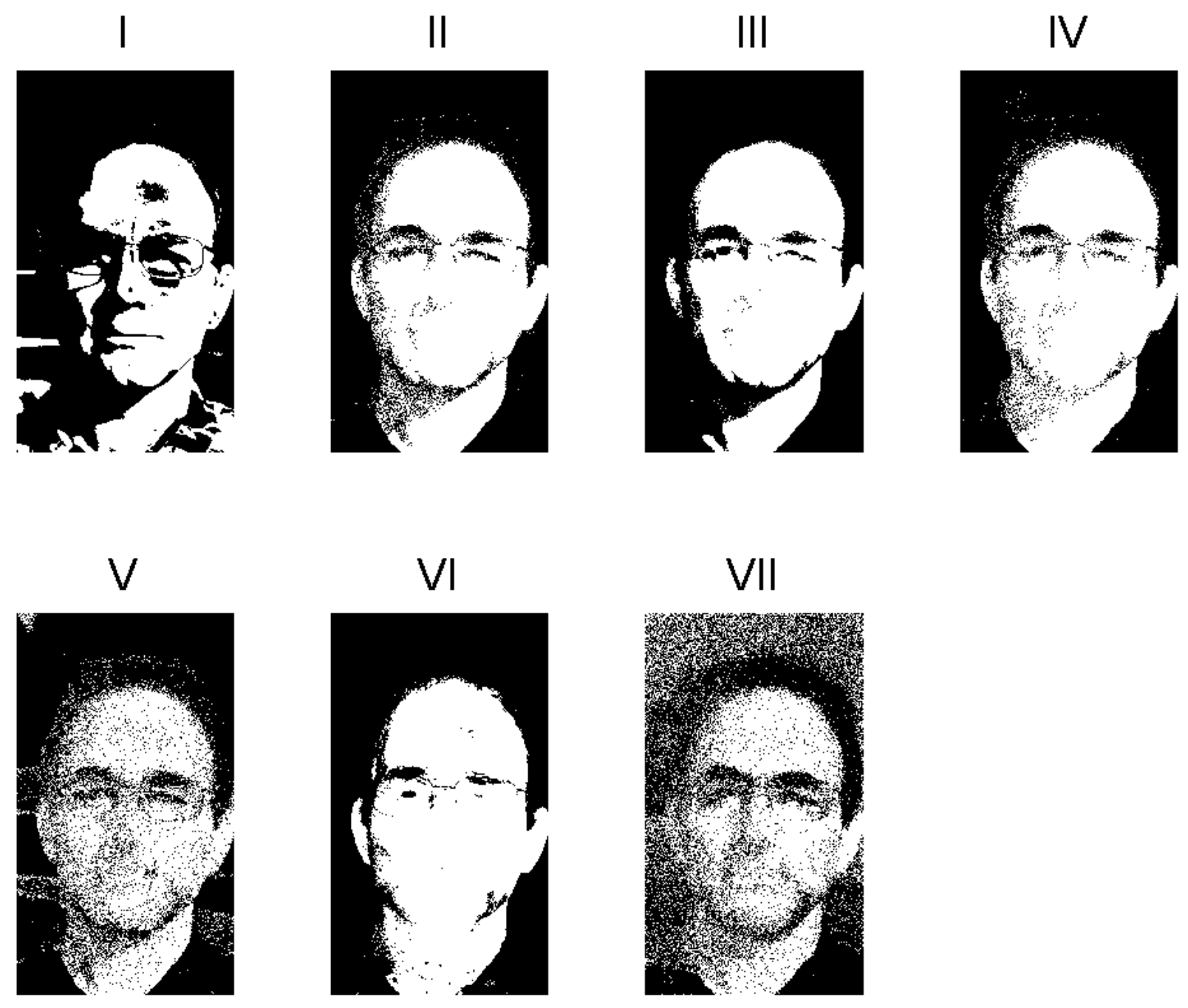

Figure 18: Binary classification of skin and non-skin: I) Full HSI cube.

II) NDGRI+NDVI+SNDI. III) NDGRI+NDVI. IV) NDGRI + SNDI. V) NDVI+SNDI. VI) NDGRI. VII) SNDI.

Once more, NDGRI had the highest overall accuracy results, based on accuracy assessment in Table 12 and the error rates in Table 13. 
Table 12: Accuracy assessment for subject-three face image.

\begin{tabular}{|c|c|c|c|c|c|c|c|c|c|c|}
\hline & & & & $\begin{array}{c}\text { All } \\
\text { Bands }\end{array}$ & $\begin{array}{c}\text { NDGRI, } \\
\text { NDVI\& } \\
\text { SNDI } \\
\end{array}$ & $\begin{array}{l}\text { NDGRI } \\
\& \text { NDVI }\end{array}$ & $\begin{array}{l}\text { NDGRI } \\
\& \text { SNDI }\end{array}$ & $\begin{array}{c}\text { NDVI \& } \\
\text { SNDI }\end{array}$ & NDGRI & SNDI \\
\hline \multirow{10}{*}{$\begin{array}{c}\text { Subject } \\
\text { Three }\end{array}$} & \multirow{5}{*}{ Test } & \multirow{2}{*}{$\begin{array}{l}\text { non- } \\
\text { skin }\end{array}$} & producer & 84.08 & 99.82 & 99.92 & 99.49 & 84.61 & 97.63 & 84.19 \\
\hline & & & user & 75.08 & 99.71 & 100.00 & 99.68 & 97.66 & 99.58 & 97.95 \\
\hline & & \multirow[t]{2}{*}{ skin } & producer & 88.45 & 99.86 & 100.00 & 99.85 & 98.77 & 99.79 & 98.91 \\
\hline & & & user & 93.07 & 99.91 & 99.96 & 99.75 & 91.34 & 98.82 & 91.03 \\
\hline & & \multicolumn{2}{|c|}{ total } & 87.17 & 99.84 & 99.97 & 99.73 & 93.41 & 99.07 & 93.30 \\
\hline & \multirow{5}{*}{ Train } & \multirow{2}{*}{$\begin{array}{l}\text { non- } \\
\text { skin }\end{array}$} & producer & 98.92 & 99.98 & 99.97 & 99.98 & 97.33 & 100.00 & 96.23 \\
\hline & & & user & 97.55 & 98.19 & 100.00 & 97.62 & 94.66 & 99.09 & 89.81 \\
\hline & & \multirow[t]{2}{*}{ skin } & producer & 91.57 & 93.85 & 99.99 & 92.08 & 82.45 & 96.81 & 70.34 \\
\hline & & & user & 96.16 & 99.93 & 99.90 & 99.92 & 90.60 & 99.99 & 87.30 \\
\hline & & \multicolumn{2}{|c|}{ total } & 97.25 & 98.57 & 99.97 & 98.12 & 93.78 & 99.28 & 89.26 \\
\hline
\end{tabular}

Table 13: Error rates for subject-three face image.

\begin{tabular}{|c|c|c|c|c|c|c|c|c|c|}
\hline & & & $\begin{array}{c}\text { All } \\
\text { Bands }\end{array}$ & $\begin{array}{c}\text { NDGRI, } \\
\text { NDVI\& } \\
\text { SNDI }\end{array}$ & $\begin{array}{l}\text { NDGRI } \\
\& \text { NDVI }\end{array}$ & $\begin{array}{l}\text { NDGRI } \\
\& \text { SNDI }\end{array}$ & $\begin{array}{c}\text { NDVI \& } \\
\text { SNDI }\end{array}$ & NDGRI & SNDI \\
\hline \multirow{4}{*}{ Subject Three } & \multirow{2}{*}{ Test } & False Positive Rate & 15.92 & 0.18 & 0.08 & 0.51 & 15.39 & 2.37 & 15.81 \\
\hline & & False Negative Rate & 11.55 & 0.14 & 0.00 & 0.15 & 1.23 & 0.21 & 1.09 \\
\hline & \multirow{2}{*}{ Train } & False Positive Rate & 1.08 & 0.02 & 0.03 & 0.02 & 2.67 & 0.00 & 3.77 \\
\hline & & False Negative Rate & 8.43 & 6.15 & 0.01 & 7.92 & 17.55 & 3.19 & 29.66 \\
\hline
\end{tabular}

\subsection{Subject-Four}

The fourth image corresponds to Subject-four. Subject-four's scene is completely different than subject-one's scene. Subject-four was placed in a grassy area sitting down next to a $99 \%$ white standard. The white standard was placed in the scene to help in the calibration of the image to reflectance units. The calibrated image was generated by averaging the spectral responses over the white standard and dividing all of the pixels in the image by that average response. Figure 19 (a) - (c) show the results of a color palette applied to each of the ratio images before calibration and Figure 19(d) shows a false RGB composite for subject-four before calibration. 
NDGRI
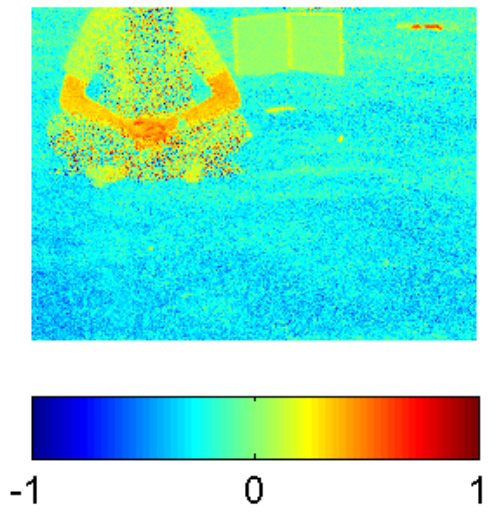

(a)

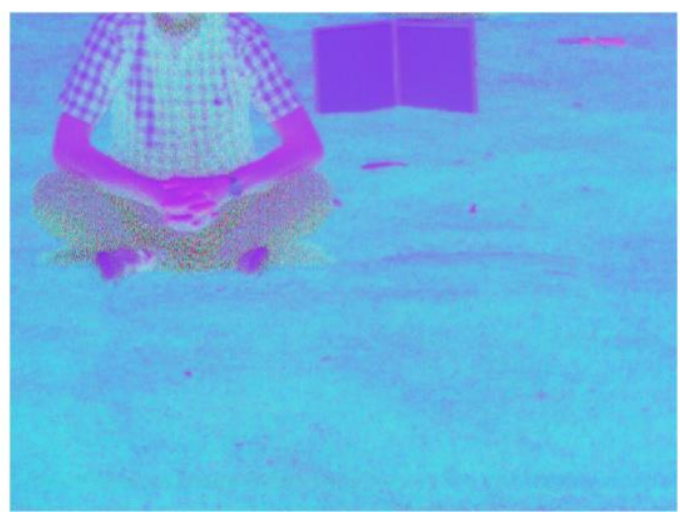

(d)

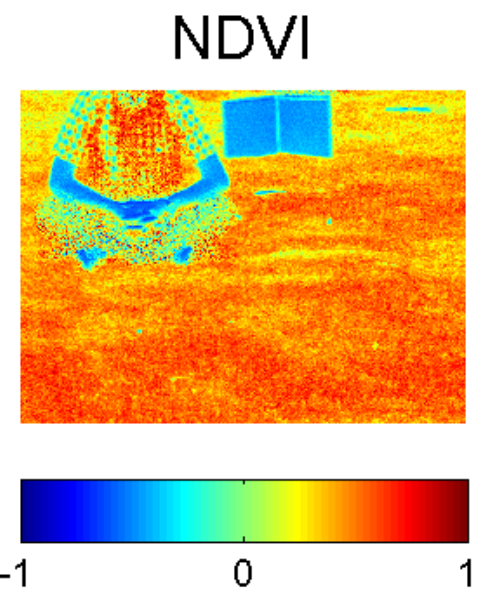

(b)
SNDI

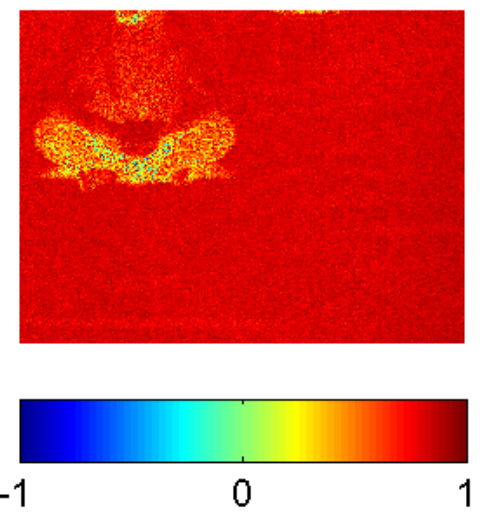

(c)

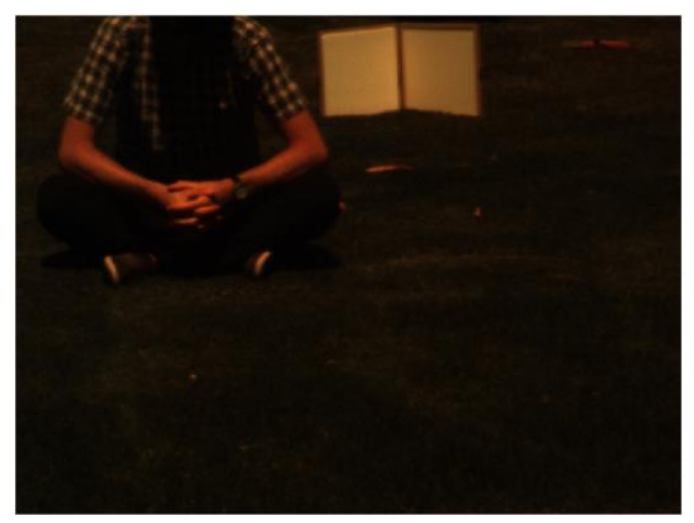

(e)

Figure 19: Color palettes for (a) NDVI, (b) NDGRI, (c) SNDI, and (d) RGB composite using RNDGRI, G-NDVI and B-SNDI. RGB composites using R 650nm, G 510nm and $\mathrm{B} \sim 475 \mathrm{~nm}$.

Figure 20 (a) - (c) show the results of a color palette applied to each of the ratio images after calibration and Figure 20(d) shows a false RGB composite for subject-four after calibration. The major difference observed between the color palettes between the calibrated and noncalibrated images is the way skin and other features are depicted. In the calibrated image the skin appears redder on NDGRI and bluer on NDVI. The grass appears a different color on all of the color palettes for all ratios in calibrated and non-calibrated images. On the RGB composite the skin appears a purple while in the calibrated image it appears fuchsia. In addition, the RGB 
composites shows how the image colors were corrected by observing the white standard which initially appeared a yellowish color in Figure 19(e) and now appears white in Figure 20(e).

NDGRI
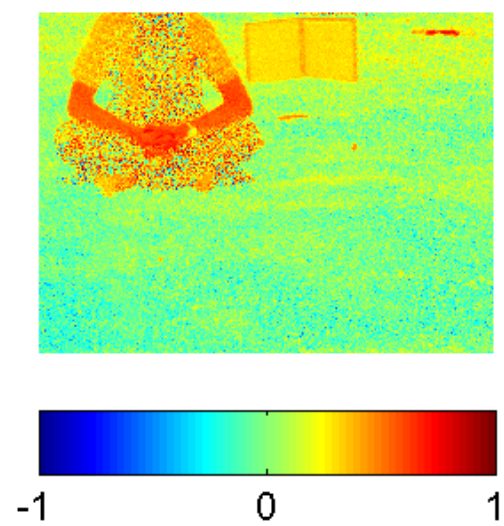

(a)

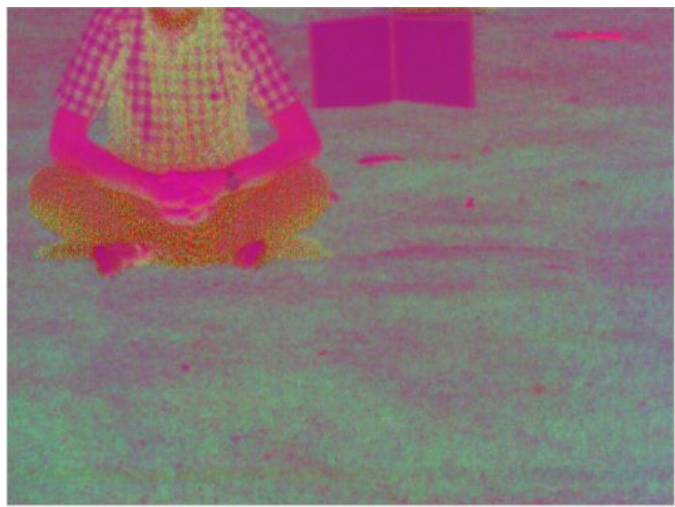

(d)
NDVI
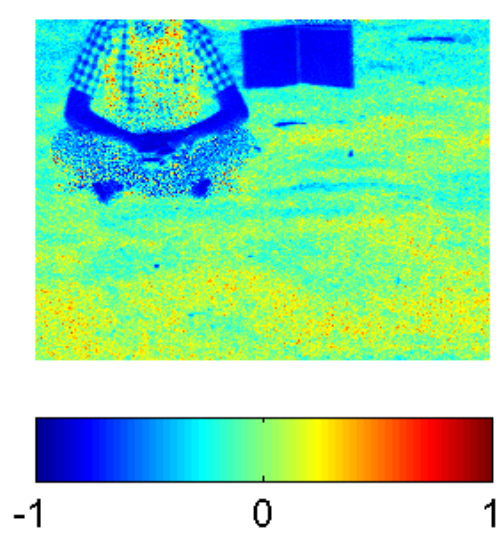

(b)
SNDI
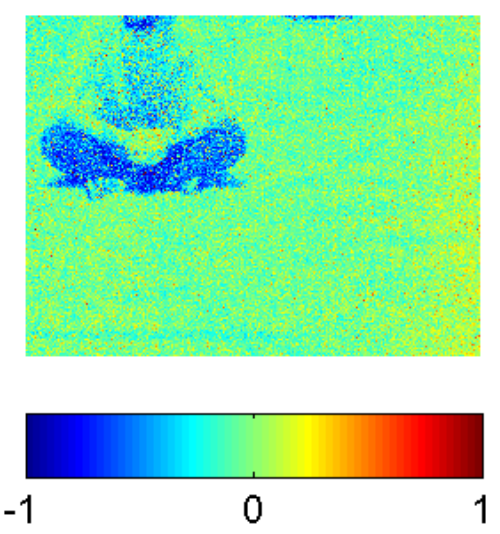

(c)

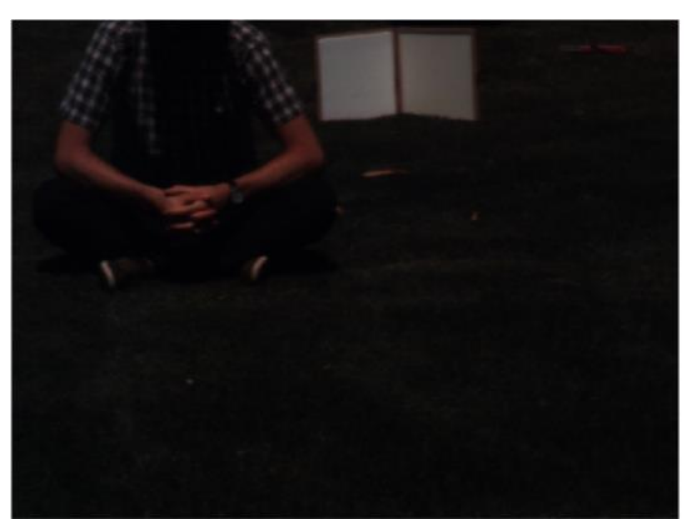

(e)

Figure 20: Color palettes for (a) NDVI, (b) NDGRI, (c) SNDI, and (d) RGB composite using RNDGRI, G-NDVI and B-SNDI. RGB composites using R 650nm, G 510nm and $\mathrm{B} \sim 475 \mathrm{~nm}$.

Classification results for all feature images in Table 3 using the training and testing samples shown in Figure 12(c) are shown in Figure 21 for Subject-four's non-calibrated image and Figure 22 for Subject-four's calibrated image. In the binary images, the white color represents the skin class and the black is the non-skin class. 


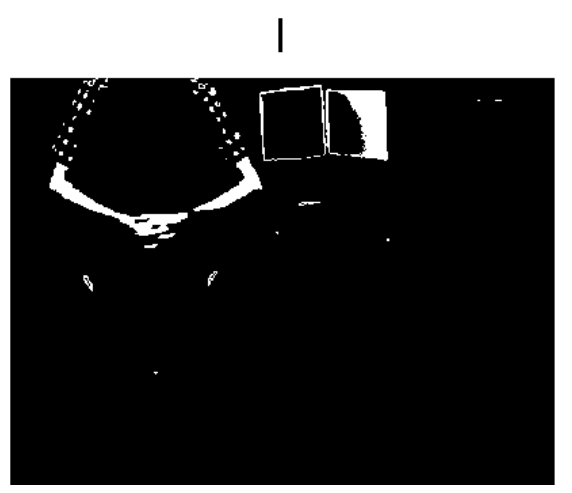

III
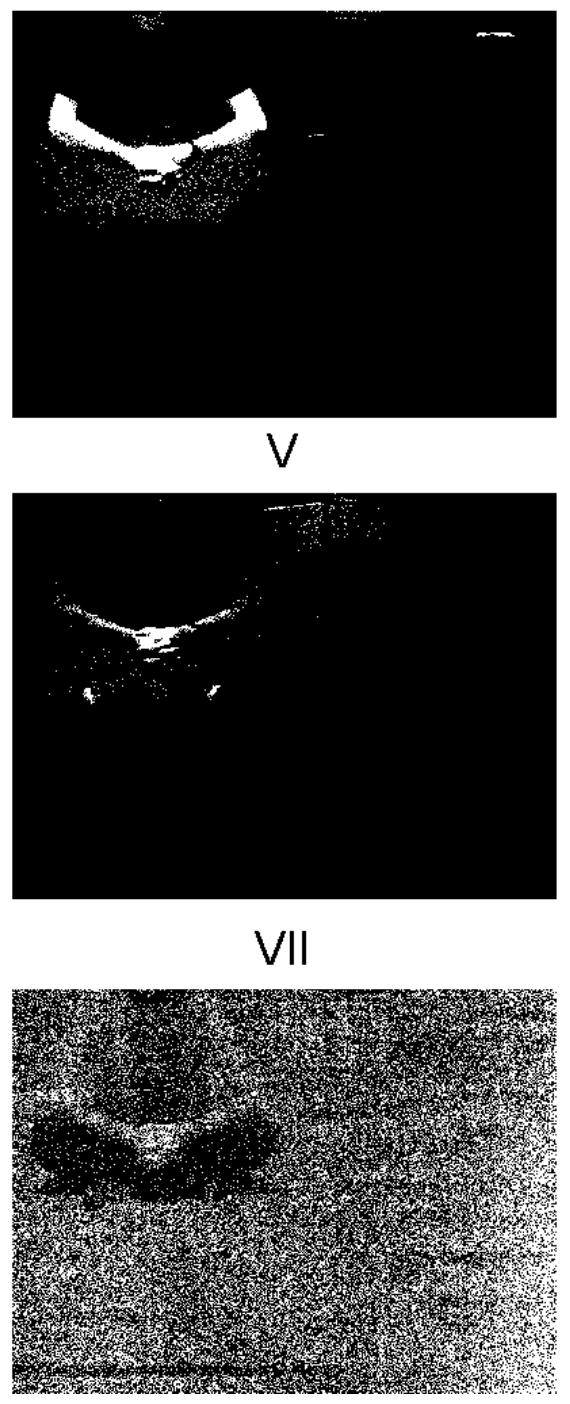

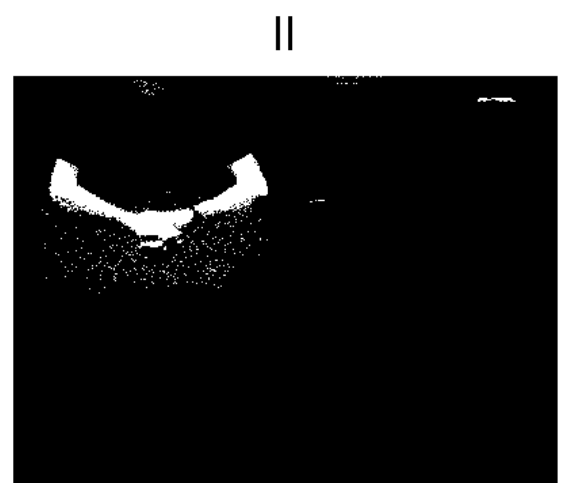

IV
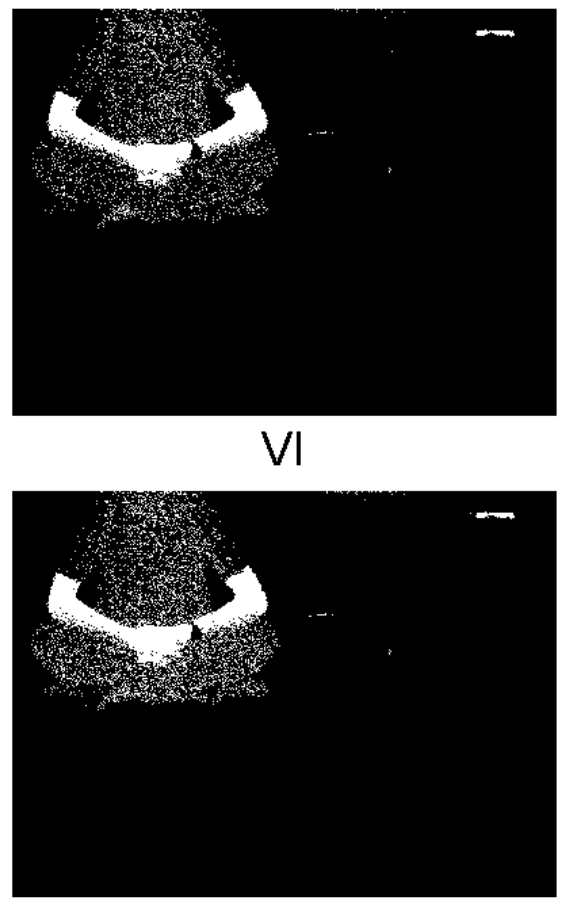

Figure 21: Binary classification of skin and non-skin: I) Full HSI cube.

II) NDGRI+NDVI+SNDI. III) NDGRI+NDVI. IV) NDGRI + SNDI. V) NDVI+SNDI. VI) NDGRI. VII) SNDI. 


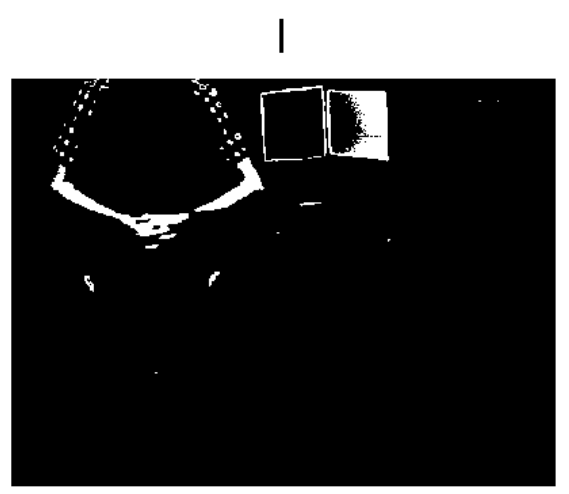

III
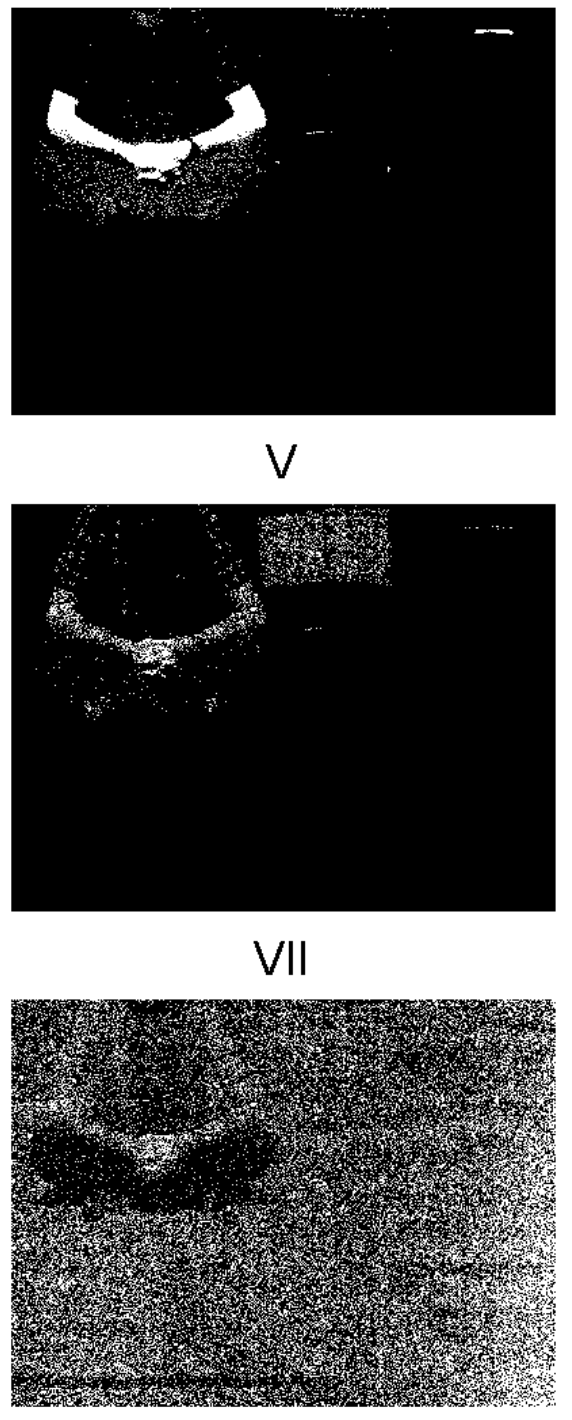

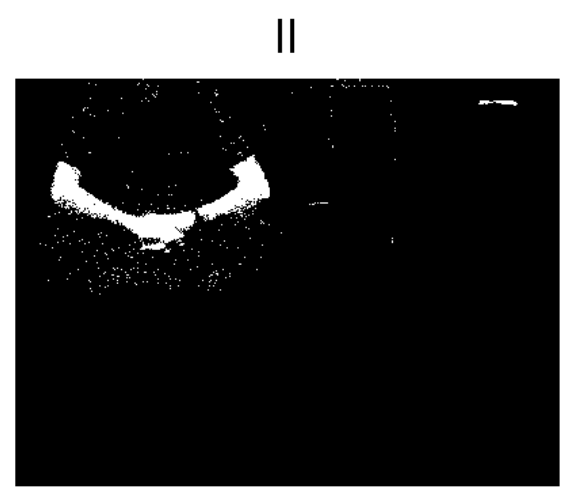

IV

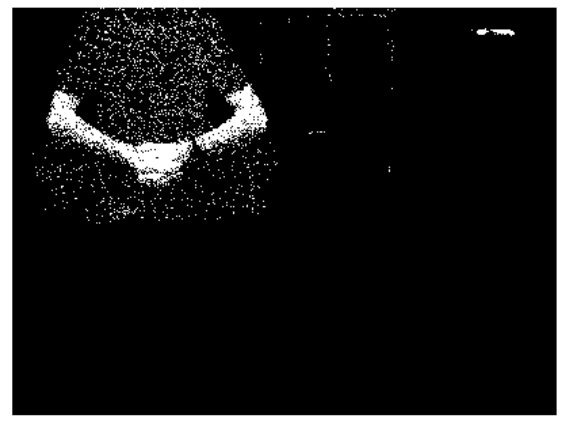

VI

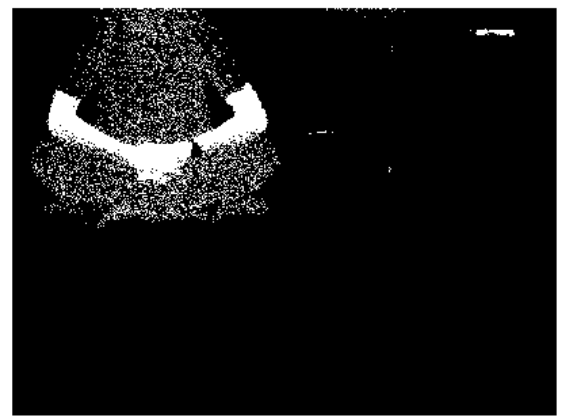

Figure 22: Binary classification of skin and non-skin: I) Full HSI cube.

II) NDGRI+NDVI+SNDI. III) NDGRI+NDVI. IV) NDGRI + SNDI. V) NDVI+SNDI. VI) NDGRI. VII) SNDI. 
Table 14 displays the accuracy assessment and Table 15 displays the Error rates for Subject-four's calibrated and non-calibrate images.

Table 14: Accuracy assessment for subject-four image.

\begin{tabular}{|c|c|c|c|c|c|c|c|c|c|c|}
\hline & & & & $\begin{array}{c}\text { All } \\
\text { Bands }\end{array}$ & $\begin{array}{c}\text { NDGRI, } \\
\text { NDVI\& } \\
\text { SNDI }\end{array}$ & $\begin{array}{l}\text { NDGRI } \\
\& \text { NDVI }\end{array}$ & $\begin{array}{l}\text { NDGRI } \\
\& \text { SNDI }\end{array}$ & $\begin{array}{c}\text { NDVI \& } \\
\text { SNDI }\end{array}$ & NDGRI & SNDI \\
\hline \multirow{10}{*}{$\begin{array}{l}\text { Subject Four } \\
\text { (Non- Cal) }\end{array}$} & \multirow{5}{*}{ Test } & \multirow{2}{*}{$\begin{array}{l}\text { non- } \\
\text { skin }\end{array}$} & producer & 98.46 & 99.93 & 99.92 & 99.97 & 95.94 & 99.98 & 95.40 \\
\hline & & & user & 99.30 & 99.81 & 99.73 & 99.04 & 99.93 & 98.57 & 59.87 \\
\hline & & \multirow[t]{2}{*}{ skin } & producer & 83.32 & 96.39 & 94.86 & 83.91 & 91.65 & 77.82 & 5.08 \\
\hline & & & user & 69.24 & 98.53 & 98.49 & 99.47 & 16.09 & 99.51 & 42.67 \\
\hline & & \multicolumn{2}{|c|}{ total } & 97.86 & 99.75 & 99.67 & 99.06 & 95.91 & 98.62 & 59.05 \\
\hline & \multirow{5}{*}{ Train } & \multirow{2}{*}{$\begin{array}{l}\text { non- } \\
\text { skin }\end{array}$} & producer & 99.99 & 100.00 & 100.00 & 100.00 & 99.95 & 100.00 & 99.65 \\
\hline & & & user & 100.00 & 99.91 & 99.85 & 99.43 & 99.80 & 99.00 & 60.35 \\
\hline & & \multirow[t]{2}{*}{ skin } & producer & 100.00 & 84.40 & 77.31 & 47.06 & 69.92 & 33.64 & 0.74 \\
\hline & & & user & 98.37 & 100.00 & 100.00 & 100.00 & 89.67 & 100.00 & 58.15 \\
\hline & & & tal & 99.99 & 99.91 & 99.85 & 99.43 & 99.75 & 99.00 & 60.34 \\
\hline \multirow{10}{*}{$\begin{array}{l}\text { Subject Four } \\
\quad(\mathrm{Cal})\end{array}$} & \multirow{5}{*}{ Test } & \multirow{2}{*}{$\begin{array}{l}\text { non- } \\
\text { skin }\end{array}$} & producer & 98.50 & 99.93 & 99.99 & 99.47 & 96.79 & 99.98 & 95.41 \\
\hline & & & user & 99.24 & 99.76 & 99.44 & 99.11 & 98.50 & 98.47 & 65.15 \\
\hline & & \multirow[t]{2}{*}{ skin } & producer & 82.31 & 95.40 & 89.91 & 83.58 & 54.05 & 76.56 & 5.16 \\
\hline & & & user & 69.91 & 98.62 & 99.78 & 89.60 & 35.02 & 99.56 & 37.69 \\
\hline & & \multicolumn{2}{|c|}{ total } & 97.84 & 99.71 & 99.45 & 98.66 & 95.46 & 98.52 & 63.83 \\
\hline & \multirow{5}{*}{ Train } & \multirow{2}{*}{$\begin{array}{l}\text { non- } \\
\text { skin }\end{array}$} & producer & 99.99 & 100.00 & 100.00 & 99.98 & 99.81 & 100.00 & 99.64 \\
\hline & & & user & 100.00 & 99.89 & 99.67 & 99.71 & 98.34 & 98.96 & 65.64 \\
\hline & & \multirow[t]{2}{*}{ skin } & producer & 100.00 & 82.51 & 60.73 & 62.90 & 16.06 & 32.86 & 0.78 \\
\hline & & & user & 98.37 & 100.00 & 100.00 & 96.74 & 62.50 & 100.00 & 53.26 \\
\hline & & \multicolumn{2}{|c|}{ total } & 99.99 & 99.89 & 99.67 & 99.69 & 98.16 & 98.97 & 65.57 \\
\hline
\end{tabular}

Table 15: Error rates for subject-four image.

\begin{tabular}{|c|c|c|c|c|c|c|c|c|c|}
\hline & & & $\begin{array}{c}\text { All } \\
\text { Bands }\end{array}$ & $\begin{array}{c}\text { NDGRI, } \\
\text { NDVI\& } \\
\text { SNDI }\end{array}$ & $\begin{array}{l}\text { NDGRI } \\
\& \text { NDVI }\end{array}$ & $\begin{array}{l}\text { NDGRI } \\
\& \text { SNDI }\end{array}$ & $\begin{array}{c}\text { NDVI \& } \\
\text { SNDI }\end{array}$ & NDGRI & SNDI \\
\hline \multirow{4}{*}{$\begin{array}{l}\text { Subject Four } \\
\text { (Non-Cal) }\end{array}$} & \multirow{2}{*}{ Test } & False Positive Rate & 1.54 & 0.07 & 0.08 & 0.03 & 4.06 & 0.02 & 4.60 \\
\hline & & False Negative Rate & 16.68 & 3.61 & 5.14 & 16.09 & 8.35 & 22.18 & 94.92 \\
\hline & \multirow{2}{*}{ Train } & False Positive Rate & 0.01 & 0.00 & 0.00 & 0.00 & 0.05 & 0.00 & 0.35 \\
\hline & & False Negative Rate & 0.00 & 15.60 & 22.69 & 52.94 & 30.08 & 66.36 & 99.26 \\
\hline \multirow{4}{*}{$\begin{array}{l}\text { Subject Four } \\
\quad(\mathrm{Cal})\end{array}$} & \multirow{2}{*}{ Test } & False Positive Rate & 1.50 & 0.07 & 0.01 & 0.53 & 3.21 & 0.02 & 4.59 \\
\hline & & False Negative Rate & 17.69 & 4.60 & 10.09 & 16.42 & 45.95 & 23.44 & 94.84 \\
\hline & \multirow{2}{*}{ Train } & False Positive Rate & 0.01 & 0.00 & 0.00 & 0.02 & 0.19 & 0.00 & 0.36 \\
\hline & & False Negative Rate & 0.00 & 17.49 & 39.27 & 37.10 & 83.94 & 67.14 & 99.22 \\
\hline
\end{tabular}


Results for Subject-four were qualitatively similar to those for subject-one in both raw (before calibration) and calibrated images. NDGRI continues to do a good job in highlighting skin by itself in all cases. However, after calibration, the exposed arms become more visible but the number of false alarms also increased as you can see from the classification result. Also, when combining all the indices, the number of false alarms decreased and skin features became clearer in the image. The accuracies reported in Table 5 confirmed that for the most part NDGRI does and excellent discrimination work by itself in the testing and training data. Accuracies may improve a bit when all three indices are combined. However, visual analysis of the classification map shows a significant reduction in false alarms for the three index combinations when compared to the NDGRI classification map. This can be significant as in a search and rescue operation we are reducing the search area by reducing the false alarms. 


\section{Chapter 5: Conclusion and Future Work}

This thesis presented preliminary results in skin detection using hyperspectral imagery. The existing NDGRI index does an excellent work by itself in discrimination between skin and non-skin classes. Accuracies in the testing and training data were similar between classifications using the NDGRI by itself and when the NDGRI is combined with the proposed SNDI and the NDVI. However, visual results from the classification maps for the entire image show that false alarms were significantly reduced when the three features were combined over classification using only the NDGRI. This is an important result since our objective is to use the indices to identify regions of interest for further scrutiny in search and rescue or surveillance operations so high probability of detection should be accompanied by a low probability of false alarms. The experimental results show that the proposed feature combination of NDGRI+NDVI+SNDI has comparable detection rates with NDGRI by itself but much lower false alarm rates. This is an encouraging result which shows that future work should be pursued.

\subsection{Conclusions}

Based on the experimental results, it may be concluded that NDGRI is the best feature for skin discrimination. This result agrees with published literature. However by combining NDGRI with other features the number of false alarms significantly decreases; this may not be directly related to the reported accuracy results in the experiments but is clearly evident in the binary classification images. As NDGRI is paired with one or more indices one may observe the facial features become clearer. When all three indices are used the best classification may be observed in all binary images. This is important as when one has a large area and skin is not clearly visible we would like to ensure that whatever pixels are being classified as skin are really skin and not

false alarms. Defense, surveillance, and search and rescue would greatly benefit from this as they would not be waiting resources on things that were wrongly classified as skin. 


\subsection{Future Work}

This research may be expended in the near future in various ways. The first way this research may be expanded is by collecting more images or having a larger database of diverse individuals (different skin colors) and backgrounds. This can help in further evaluating the usefulness of the proposed 3-ratio combination using the classification analysis.

In addition, developing a better understanding of atmospheric effects in ratio performance will be important as we observed that SNDI was affected by the atmospheric transmissivity as there is a water absorption band near the $980 \mathrm{~nm}$ feature. Notice that the contrast between background and skin with this index was not as high as with NDGRI.

Another way this research may be expanded is to identify threshold values that can be applied to the normalized difference indices images for operational purposes. Once a set of consistent thresholds is found one could move on to create a skin detector which would mean one could utilize this algorithm at real time speeds. 


\section{References}

[1] Elgammal, A., Muang, C., \& Hu, D. (2009). Skin detection. In Li, S.Z. (Ed.), Encyclopedia of Biometrics (pp. 1218-1224). New York: Springer.

[2] Borengasser, M., \& Hungate, W. (2008). Hyperspectral remote sensing: Principles and applications (pp. 1-6). Boca Raton, FL: CRC Press.

[3] Ambinder, M. (2011, May 2). The Secret Team That Killed bin Laden. Retrieved May 2, 2015, from http://www.nationaljournal.com/whitehouse/the-secret-team-that-killed-binladen-20110502

[4] Velez-Reyes, M., Goodman, J.A., \& Saleh, B.E., (2011). Chapter 6: Spectral Imaging. In Saleh, B.E. (Ed.), Introduction to subsurface imaging (pp. 231-273). Cambridge: Cambridge University Press.

[5] Eismann, M.T. (2012). Hyperspectral remote sensing. Bellingham, Wash. (1000 20th St. Bellingham WA 98225-6705 USA): SPIE.

[6] Elowitz, R.M. (2009). Figure 3: Hyperspectral imaging, also known as chemical sensing, allows for the differentiation, display and classification of objects based on their chemical composition. Retrieved December 5, 2014 from http://www.nature.com/nphoton/journal/v3/n11/images/nphoton.2009.205-f3.jpg

[7] [Untitled illustration of a spaceborne hyperspectral sensor]. Retrieved December 5, 2014 from http://cropmetrics.com/wp-content/uploads/mit.png

[8] Ashraf, M.A., Maah, J., \& Yusoff, I., (2011). Introduction to Remote Sensing of Biomass. In, Dr. Atazadeh I. (Ed.), Biomass and Remote Sensing of Biomass. ISBN: 978-953-307490-0, InTech, DOI: 10.5772/16462. Available from: http://www.intechopen.com/books/biomass-and-remote-sensing-of-biomass/introductionto-remote-sensing-of-biomass

[9] National Aeronautics and Space Administration, Science Mission Directorate. (2015). Reflected Near-Infrared Waves. Retrieved May 13, 2015, from Mission: Science website: http://missionscience.nasa.gov/ems/08_nearinfraredwaves.html

[10] Chlorophyll | biology. (Sep 07, 2011). In Encyclopedia Britannica Online. Retrieved May 2, 2015, from http://www.britannica.com/EBchecked/topic/113725/chlorophyll

[11] [Untitled illustration of vegetation reflectance]. Retrieved December 5, 2014 from http://1.bp.blogspot.com/-

nO4k4eKS9fw/Uluc2cHxoVI/AAAAAAAABJE/xBPMrAavew4/s640/nir_vegetation_gr aph.gif

[12] Stratum corneum / anatomy. (n.d.). In Encyclopedia Britannica Online. Retrieved May 2, 2015, from http://www.britannica.com/EBchecked/topic/568457/stratum-corneum

[13] Melanosomes. (2003) McGraw-Hill Dictionary of Scientific \& Technical Terms, 6E. Retrieved May 2, 2015, from http://encyclopedia2.thefreedictionary.com/Melanosomes

[14] Melanin | biological pigment. (2014, Nov 17). In Encyclopedia Britannica Online. Retrieved May 2, 2015, from http://www.britannica.com/EBchecked/topic/373722/melanin 
[15] Hemoglobin | biochemistry. (2009, Jan 14). In Encyclopedia Britannica Online. Retrieved May 2, 2015, from http://www.britannica.com/EBchecked/topic/260923/hemoglobin

[16] Heather Brannon, MD (2014, December 19). Skin anatomy. Retrieved May 2, 2015, from http://dermatology.about.com/cs/skinanatomy/a/anatomy.htm

[17] Heather Brannon, MD (2014, April 22). Epidermis Anatomy. Retrieved May 2, 2015, from http://dermatology.about.com/od/anatomy/ss/epidermis.htm

[18] Heather Brannon, MD (2014, January 01). Stratum Corneum Anatomy - The Key to Healthy, Attractive Skin. Retrieved May 2, 2015, from http://dermatology.about.com/od/anatomy/ss/sc_anatomy.htm

[19] Jacques, S. (1998, January). Skin Optics. Retrieved May 2, 2015, from http://omlc.org/news/jan98/skinoptics.html

[20] [Untitled image of how skin coloration arises]. Retrieved May 2, 2015, from https://www.cs.bham.ac.uk/ exc/Research/EPSRC_skin/epsrc_skin_project.html

[21] Douma, M., curator. (2008). Humans | Plants and Animals. In Cause of Color. Retrieved May 2, 2015, from http://www.webexhibits.org/causesofcolor/3.html.

[22] Uto, K., Kosugi, Y., Murase, T., \& Takagishi, S. (2012). "Hyperspectral band selection for human detection," Sensor Array and Multichannel Signal Processing Workshop (SAM), 2012 IEEE 7th , vol., no., pp.501,504, 17-20 June 2012, DOI: 10.1109/SAM.2012.6250550

[23] Brooks, A.L. (2015). Improved Multispectral Skin Detection and its Applications to Search Space Reduction for Dismount Detection Based on Histograms of Oriented Gradients (Master's Thesis) Air Force Institute of Technology, Wright-Patterson AFB.

[24] Nunez, A.S., \& Mendenhall, M.J. (2008)."Detection of Human Skin in Near Infrared Hyperspectral Imagery," Proc 2008 IEEE Geoscience and Remote Sensing Symposium Vol.2, II-621-II-624.

[25] Nixon, K., Rowe, R., \& Allen, J. (2004). "Novel Spectroscopy-based technology for biometric and liveness verification", Proc. SPIE 5404, 287-295.

[26] Vezhnevets, V., Sazonov, V., \& Andreeva, A. (2003). A Survey on Pixel-Based Skin Color Detection Techniques, In ICCGV, pp.85-92

[27] Trierscheid, M., Pellenz, J., Paulus, D., \& Balthasar, D. (2008). "Hyperspectral Imaging or Victim Detection with Rescue Robots," Safety, Security and Rescue Robotics, SSRR 2008. IEEE International Workshop on , vol., no., pp.7,12, 21-24 Oct. 2008 DOI: 10.1109/SSRR.2008.4745869

[28] Bersha K.S. (2015). Spectral Imaging and Analysis of Human Skin (Master's Thesis) University of Eastern Finland, Joensuu, Finland.

[29] Nunez, A.S. (2015). A Physical Model of Human Skin and Its Application for Search and Rescue (Doctoral dissertation), Air Force Institute of Technology, Wright-Patterson AFB.

[30] Rosario-Torres, S., Velez-Reyes, M., Hunt, S.D., \& Jimenez, L.O. (2007). "New Developments and Application of the UPRM MATLAB Hyperspectral Image Analysis Toolbox", Proc. SPIE 6565. 
[31] Schowengerdt, R. (2007). Remote sensing models and methods for image processing (3rd ed.). Burlington, MA: Academic Press.

[32] Jensen, J.R. (1986). Introductory Digital Image Processing (pp. 379). Prentice-Hall, Englewood Cliffs, New Jersey.

[33] Landgrebe, D. A. (2003). Signal Theory Methods in Multispectral Remote Sensing, John Wiley \& Sons. 


\section{Vita}

Stephanie Sanchez earned her Bachelor of Science in Electrical Engineering from the University of Texas at El Paso (UTEP) in fall 2012. She is currently pursuing a Master of Science Degree in Electrical Engineering also at UTEP. While pursuing undergraduate studies, she contributed to various research groups. One of her contributions was creating Rappture tools and iApplications to facilitate basic electrical engineering and devices concepts in an interactive manner under the direction of Dr. Stella Quiñones. She has participated in an Undergraduate Research Fellowship at Purdue University. In this fellowship, she contributed to the improvement of the PVpanelSim tool to simulate and model the realistic behavior of photovoltaic devices under the supervision of Dr. Lundstrom and Dr. Alam. She also conducted research under the guidance and supervision of Dr. Ricardo Von Borries and Dr. Berenice Verdin. This research included running simulations on the Digital Imaging and Remote Sensing Image Generation (DIRSIG) using Light Detection And Ranging (LIDAR) sensing technology to examine the topology of an area using signal processing, more specifically a tree's characteristics in a forest. Her thesis was

focused on hyperspectral imaging and skin optics using signal processing, under the direction of Dr. Miguel Velez-Reyes. This research was presented at eth 2014 SHPE and 2015 SPIE DSS conferences. At the SHPE conference her poster placed first among the graduate poster competition. In addition, she is the author to a paper that is currently under review for the 2015 SPIE DSS conference.

Permanent address: 13534 Inspiration Ct.

Horizon City, TX, 79928

This thesis/dissertation was typed by Stephanie Michelle Sanchez. 